\title{
Probing lepton flavor violating decays in MSSM with non-holomorphic soft terms
}

\author{
Utpal Chattopadhyay, ${ }^{a}$ Debottam Das ${ }^{b, c}$ and Samadrita Mukherjee ${ }^{a}$ \\ ${ }^{a}$ School of Physical Sciences, Indian Association for the Cultivation of Science, \\ $2 A$ \& B Raja S.C. Mullick Road, Jadavpur, Kolkata 700 032, India \\ ${ }^{b}$ Institute of Physics, \\ Bhubaneswar, Odisha 751 005, India \\ ${ }^{c}$ Homi Bhabha National Institute, Training School Complex, \\ Anushakti Nagar, Mumbai 400 085, India \\ E-mail: tpuc@iacs.res.in, debottam@iopb.res.in, tpsm9@iacs.res.in
}

Abstract: The Minimal Supersymmetric Standard Model (MSSM) can be extended to include non-holomorphic trilinear soft supersymmetry (SUSY) breaking interactions that may have distinct signatures. We consider non-vanishing off-diagonal entries of the coupling matrices associated with holomorphic (of MSSM) and non-holomorphic trilinear terms corresponding to sleptons with elements $A_{i j}^{l}$ and $A_{i j}^{\prime l}$. We first improve the MSSM charge breaking minima condition of the vacuum to include the off-diagonal entries $A_{i j}^{l}$ (with $i \neq j$ ). We further extend this analysis for non-holomorphic trilinear interactions. No other sources of lepton flavor violation like that from charged slepton matrices are considered. We constrain the interaction terms via the experimental limits of processes like charged leptons decaying with lepton flavor violation (LFV) and Higgs boson decaying to charged leptons with LFV. Apart from the leptonic decays we compute all the three neutral LFV Higgs boson decays of MSSM. We find that an analysis with non-vanishing $A_{e \mu}^{\prime}$ involving the first two generations of sleptons receives the dominant constraint from $\mu \rightarrow e \gamma$. On the other hand, $A_{e \tau}^{\prime}$ or $A_{\mu \tau}^{\prime}$ can be constrained from the CMS $13 \mathrm{TeV}$ analysis giving limits to the respective Yukawa couplings via considering SM Higgs boson decaying into $e \tau$ or $\mu \tau$ final states. Contributions from $A_{i j}^{l}$ is too little to have any significance compared to the large effect from $A_{i j}^{\prime l}$.

KEywords: Supersymmetry Phenomenology

ArXIV EPrINT: 1911.05543 


\section{Contents}

1 Introduction 1

2 Theoretical framework 4

3 Analysis of charge breaking minima $\quad 9$

$\begin{array}{ll}3.1 \text { Charge breaking with flavor violation in MSSM } & 10\end{array}$

$\begin{array}{lll}3.2 & \text { Charge breaking condition in NHSSM } & 12\end{array}$

4 Status of different LFV decays 13

5 Results $\quad \mathbf{1 5}$

$\begin{array}{lll}5.1 l_{j} \rightarrow l_{i} \gamma & 17\end{array}$

$\begin{array}{lll}5.2 l_{j} \rightarrow 3 l_{i} & 17\end{array}$

$5.3 \phi(h, H, A) \rightarrow l_{i} \bar{l}_{j} \quad 19$

5.4 Direct constraints on $Y_{i j}$ from LFV Higgs decay limits of LHC 21

5.4.1 Results for varying bino and slepton masses 22

$\begin{array}{ll}\text { 5.4.2 Results for fixed bino and slepton masses } & 23\end{array}$

6 Conclusion $\quad 26$

\section{Introduction}

The Higgs data is gradually drifting towards the Standard Model (SM) expectations [1] ever since the first observation of a new resonance at the Large Hadron Collider (LHC) $[2,3]$ in 2012. Still, SM is far away to be a complete description of particle physics in view of many theoretical aspects and a few experimental data. Indeed, the unknown new physics (NP) has always been the driving force in studying particle physics for many decades to explain e.g., the existence of dark matter, neutrino masses, or matter-anti matter asymmetries. Most of these NP models offer new particles with new interactions that could possibly be tested at the LHC. Apart from this direct test, one can hope to find the NP signatures via indirect search experiments involving flavor physics, e.g., through the dedicated experiments that search for quark or charged lepton flavor violations (cLFV) like $b \rightarrow s \gamma$ or $\mu \rightarrow e \gamma$. Among the flavor violating observables, the cLFV processes are of particular interest. The reason is that in the context of Standard Model (SM) or in the minimal extension of the SM that includes the Yukawa interactions in the neutral lepton sector, the decay rates involving cLFV processes are strongly suppressed (e.g., $B R(\mu \rightarrow e \gamma) \sim 10^{-55}$ ) [4]. This can be attributed to the tinyness of the neutrino masses which is the only source of cLFV processes. However, any extension of SM, mainly in the leptonic sector may offer new particles or new interactions with the SM leptons. This can potentially change the 
cLFV decay rates drastically [5, 6] (for a review see for instance [7]). The Minimal Supersymmetric Standard Model (MSSM), the supersymmetric extension of SM with the two Higgs doublets in general may have a large number of flavor violating couplings through soft SUSY breaking interactions [8-16]. The lepton sector is particularly important in this context. Interestingly, the flavor violating soft SUSY breaking parameters may also be generated radiatively. In fact, guided by the origin of cLFV processes, one may broadly classify a few MSSM and beyond the MSSM scenarios as follows:

- In the extensions of MSSM, one may connect the origin of the cLFV to the masses for neutrinos, the existence of which have been strongly established through neutrino oscillation experiments $[17,18]$. One of the most attractive possibilities would be to consider a seesaw mechanism [19-32] that can also be generalized in the framework of SUSY models (i.e. the SUSY seesaw) [33, 34]. In the simplest example i.e, type-I SUSY seesaw, the right handed neutrino Yukawa couplings that generate neutrino masses, may also radiatively induce the SUSY soft-breaking left handed slepton mass matrices $\left(M_{\tilde{L}_{i j}}^{2}\right)$, leading to flavor violations at low energies [35-38]. This can substantially influence lepton flavor violating decays of the types $\left.\left(l_{j} \rightarrow l_{i} \gamma\right)\right)$ or three-body lepton decays $l_{j} \rightarrow 3 l_{i}$ through photon, $Z$ or Higgs penguins and the flavor violating decays of the Higgs scalars (see e.g., [36-61]). Other important probes are semileptonic $\tau$-decays, $\mu-e$ conversion of nucleus etc. The flavor changing processes involving Higgs scalars potentially become large for large $\tan \beta[42,49]$. However here the typical mass scales of the extra particles (such as right handed neutrinos) are in general very high, often close to the gauge coupling unification scale. An attractive alternative is the inverse seesaw scenario, where the presence of comparatively light right-handed neutrinos and sneutrinos can enhance the flavor violating decays [6267]. In addition to generating $M_{\tilde{L}_{i j}}^{2}$ radiatively, the right handed neutrino extended models may as well be embedded in grand unified theory (GUT) framework [68-71].

- Though neutrino mass models, particularly in presence of new states imply cLFV, the later does not necessarily imply neutrino mass generation. The simplest example is the R-parity conserving MSSM. Here direct sources of flavor violation are in the off-diagonal soft terms of the slepton mass matrices and trilinear coupling matrices (see e.g., [13-15])(specifically through $M_{\tilde{e}}^{2}, A_{f}$ ). One may probe the nonzero off-diagonal elements of all the soft SUSY breaking terms $\left(A_{f}, M_{\tilde{L}}^{2}, M_{\tilde{e}}^{2}\right)$ which may induce cLFV processes through loops mediated by sleptons-neutralinos and/or sneutrinos-charginos. This can also be realized in a High scale SUSY breaking model, e.g., in a supergravity or superstring inspired scenarios, where non-universal soft terms can be realized in the high-scale effective Lagrangian (see, for example Ref:[72] and references therein) apart from running via Renormalization Group (RG) evolution that itself may generate flavor violation $[13-15] .{ }^{1}$

\footnotetext{
${ }^{1}$ We also note in passing that intricacies related to the large number of soft breaking parameters in the cLFV computations and also the inter-generation mixings in the general MSSM can be evaded completely in a High scale SUSY model where SUSY breaking is communicated in a flavor blind manner [13-15].
} 
Although, in general, cLFV processes through radiatively induced $M_{\tilde{L}}^{2}$ may look more appealing, in some cases it may be somewhat restrictive in obtaining any significant amount of flavor violating branching ratios. On the contrary, being free from the constraints of the masses of neutrinos, soft SUSY breaking parameters can lead to reasonably large decay rates which may be interesting and could be testable in near future. In this analysis, we would go one step further. We would limit ourselves within the MSSM field content augmented by most general soft SUSY breaking terms without considering their high scale origin. In its generic form MSSM includes only holomorphic trilinear soft SUSY breaking terms [8-15]. However, in a most general framework, it has been shown that certain non-holomorphic supersymmetry breaking terms may qualify as soft terms when there is no gauge singlet field present [73-77]. Such a consideration can be phenomenologically interesting. For example, if MSSM soft SUSY breaking sector is extended to include $A_{f}^{\prime} \phi^{2} \phi^{*}$ type of interactions, one may find that an SM like CP even Higgs boson with mass $\sim 125 \mathrm{GeV}$ can be achieved with relatively lighter squarks with the help of the specific $A_{t}^{\prime}$ [78], the relevant coupling from non-holomorphic trilinear interaction. Similarly, the non-holomorphic (NH) terms may also be helpful to fulfill constraints from rare B-decays (viz. $\operatorname{Br}\left(B \rightarrow X_{s} \gamma\right), \operatorname{Br}\left(B_{s} \rightarrow \mu^{+} \mu^{-}\right)$etc) both in a phenomenological MSSM (pMSSM) like scenario [78] or in some high scale model like constrained MSSM (CMSSM) [79-81] or minimal Gauge Mediated Supersymmetry Breaking (mGMSB) [82]. Another interesting feature is that a small NH trilinear coupling (namely $A_{\mu}^{\prime}$ ) may be capable to attune the inflexible constraints of $(g-2)_{\mu}$ [78]. Focusing only on the leptonic sector, the playground associated with the NH soft terms may not be completely free, rather there can be strong constraints appearing from different lepton flavor violating decays via their off-diagonal entries [83]..$^{2}$ This is of-course similar to holomorphic trilinear interactions of MSSM, apart from an enhancement by $\tan \beta$ with $A_{i}^{\prime}$ associated with down type of quark and lepton. We will consider the slepton mass squared matrix to be diagonal, and consider that the only source of cLFV to be the holomormorphic and non-holomorphic trilinear coupling matrices, namely $A_{f} \& A_{f}^{\prime}$. For the sake of explicit understanding we will scan either $A_{f}$ or $A_{f}^{\prime}$ at a time to find the allowance of associated off-diagonal elements under the present and future experimental sensitivities of different cLFV observables. In order to perform this analysis, a more important checkpoint is avoidance of any dangerous charge and color breaking global minima (CCB). It is known that a large trilinear coupling (diagonal) in general, lead to unphysical or metastable CCB minima. For lepton flavor it is only the charge breaking $(\mathrm{CB})$ of vacuum that is of concern, but it requires the involvement of the off-diagonal entries of the trilinear couplings. In this analyses we will necessarily improve the charge breaking constraint for a general trilinear coupling matrix having non-vanishing entries in its diagonal and off-diagonal elements. So, we should survey if there is any violation in charge breaking minima condition and always ensure that the electroweak symmetry breaking minimum i.e., the global minimum is a charge conserving one.

Popular examples are mSUGRA, anomaly-mediated supersymmetry breaking (AMSB) or gauge-mediated supersymmetry breaking (GMSB).

${ }^{2}$ For chirally-enhanced flavor violating loop processes with most general set of soft terms see [84]. 
The rest of the work is ordered as follows. In section 2 we discuss the theoretical framework that includes the slepton mass matrices in presence of the $\mathrm{NH}$ terms along with the cLFV observables which we would consider in the analysis. Section 3 covers the examination of analytical structure of charge breaking minima in the context of our model. Numerical results are presented in section 4. Here we take the inter-generational mixings in the trilinear couplings of the slepton fields for both holomorphic and non-holomorphic couplings. The free rise of these couplings are limited via charge breaking minima and also from the non-observation of any signals at the LHC apart from the same experiments that search for cLFV processes. Here we also consider the future sensitivity of the experiments. In closing we conclude in section 5 .

\section{Theoretical framework}

We focus on the general lepton flavor mixing through left and right slepton mixing in the MSSM with R-parity conserved. We will further include contributions from non-standard soft supersymmetry breaking interactions. The superpotential is given by,

$$
W_{M S S M}=\bar{U} \mathbf{y}_{\mathbf{u}} Q \cdot H_{u}-\bar{D} \mathbf{y}_{\mathbf{d}} Q \cdot H_{d}-\bar{E} \mathbf{y}_{\mathbf{e}} L \cdot H_{d}+\mu H_{u} \cdot H_{d}
$$

where $y$ 's are the Yukawa matrices in flavor space. The MSSM soft terms read [11-15],

$$
\begin{aligned}
-\mathcal{L}_{\mathrm{soft}}^{M S S M}= & \frac{1}{2}\left(M_{3} \tilde{g} \tilde{g}+M_{2} \tilde{W} \tilde{W}+M_{1} \tilde{B} \tilde{B}+c . c\right) \\
& +\left(\tilde{u}_{i R}^{*} \mathbf{A}_{\mathbf{u} i j} \tilde{q}_{j L} \cdot H_{u}+\tilde{d}_{i R}^{*} \mathbf{A}_{\mathbf{d} i j} \tilde{q}_{j L} \cdot H_{d}+\tilde{e}_{i R}^{*} \mathbf{A}_{\mathbf{l} i j} \tilde{\ell}_{j L} \cdot H_{d}+h . c .\right) \\
& +\tilde{q}_{i L}^{\dagger} \mathbf{M}_{\tilde{\mathbf{q}}_{i j}}^{2} \tilde{q}_{j L}+\tilde{\ell}_{i L}^{\dagger} \mathbf{M}_{\tilde{\mathbf{L}} i j}^{2} \tilde{\ell}_{j L}+\tilde{\tilde{u}}_{i R}^{*} \mathbf{M}_{\tilde{\mathbf{u}} i j}^{2} \tilde{u}_{j R}^{* \dagger}+\tilde{d}_{i R}^{*} \mathbf{M}_{\tilde{\mathbf{d}} i j}^{2} \tilde{d}_{j R}^{* \dagger} \\
& +\tilde{e}_{i R}^{*} \mathbf{M}_{\tilde{\mathbf{e}} i j}^{2} \tilde{e}_{j R}^{* \dagger}+m_{H_{u}}^{2} H_{u}^{*} H_{u}+m_{H_{d}}^{2} H_{d}^{*} H_{d}+\left(B_{\mu} H_{u} \cdot H_{d}+c . c\right) .
\end{aligned}
$$

MSSM can be extended to include a set of possible additional non-holomorphic trilinear soft SUSY breaking terms as given below [73-75],

$$
-\mathcal{L}_{\text {soft }}^{\prime N H} \supset \tilde{u}_{i R}^{*} \mathbf{A}_{\mathbf{u} i j}^{\prime} \tilde{q}_{j L} \cdot H_{d}^{*}+\tilde{d}_{i R}^{*} \mathbf{A}_{\mathbf{d} i j}^{\prime} \tilde{q}_{j L} \cdot H_{u}^{*}+\tilde{e}_{i R}^{*} \mathbf{A}_{\mathbf{e} i j}^{\prime} \tilde{l}_{j L} \cdot H_{u}^{*}+h . c . .
$$

Here $i, j=1,2,3$ denote indices in the fermion (f) family space. In contrast to eq. (2.2) here the sfermions $\tilde{u}_{i R}^{*}$ and $\tilde{q}_{i L}$ couple with $H_{d}^{*}$ instead of $H_{u}$. The trilinear coupling matrices are typically scaled and characterized by the quark/charged lepton Yukawa couplings. The effect of above non-standard or non-holomorphic terms (eq. (2.3)) are reflected in the mass matrices and mixing angles of physical sparticles. Since we are concerned with general flavor mixing in the slepton sector, the general form of $6 \times 6$ slepton mass squared matrix is written in the electroweak basis $\left(\tilde{e}_{L}, \tilde{\mu}_{L}, \tilde{\tau}_{L}, \tilde{e}_{R}, \tilde{\mu}_{R}, \tilde{\tau}_{R}\right)$ in terms of left and right handed blocks as given below.

$$
M_{\tilde{l}}^{2}=\left(\begin{array}{ll}
M_{\tilde{l} L L}^{2} & M_{\tilde{l} L R}^{2} \\
M_{\tilde{l} R L}^{2} & M_{\tilde{l} R R}^{2}
\end{array}\right)
$$


In the above, each block is a $3 \times 3$ matrix where one has,

$$
\begin{aligned}
& M_{\tilde{l} L L_{i j}}^{2}=M_{\tilde{L}_{i j}}^{2}+\left(M_{Z}^{2}\left(-\frac{1}{2}+\sin ^{2} \theta_{W}\right) \cos 2 \beta+m_{\ell_{i}}^{2}\right) \delta_{i j}, \\
& M_{\tilde{l} R R_{i j}}^{2}=M_{\tilde{e}_{i j}}^{2}+\left(-M_{Z}^{2} \sin ^{2} \theta_{W} \cos 2 \beta+m_{\ell_{i}}^{2}\right) \delta_{i j} .
\end{aligned}
$$

Here $\beta$ is defined via $\tan \beta=\frac{v_{u}}{v_{d}}$, the ratio of Higgs vacuum expectation values. $\theta_{W}$ and $M_{Z}$ refer to the Weinberg angle and the Z-boson mass respectively whereas $m_{\ell_{i}}$ refers to lepton masses respectively. The non-holomorphic trilinear couplings modify slepton left-right mixings. For MSSM with non-holomorphic soft terms one has the following. ${ }^{3}$

$$
\begin{aligned}
M_{\tilde{l} L R}^{2} & =\left(\begin{array}{ccc}
\left(A_{e}-\left(\mu+A_{e}^{\prime}\right) \tan \beta\right) & A_{e \mu}-A_{e \mu}^{\prime} \tan \beta & A_{e \tau}-A_{e \tau}^{\prime} \tan \beta \\
A_{\mu e}-A_{\mu e}^{\prime} \tan \beta & \left(A_{\mu}-\left(\mu+A_{\mu}^{\prime}\right) \tan \beta\right) & A_{\mu \tau}-A_{\mu \tau}^{\prime} \tan \beta \\
A_{\tau e}-A_{\tau e}^{\prime} \tan \beta & A_{\tau \mu}-A_{\tau \mu}^{\prime} \tan \beta & \left(A_{\tau}-\left(\mu+A_{\tau}^{\prime}\right) \tan \beta\right)
\end{array}\right) \\
M_{\tilde{l} R L}^{2} & =\left(M_{\tilde{l} L R}^{2}\right)^{\dagger} .
\end{aligned}
$$

With only three sneutrino eigenstates, $\tilde{\nu}_{L}$ with $\nu=\nu_{e}, \nu_{\mu}, \nu_{\tau}$ in MSSM, the sneutrino mass matrix corresponds to a $3 \times 3$ matrix. We note that the non diagonality in flavor comes exclusively from the soft SUSY-breaking parameters. The main non-vanishing sources for $i \neq j$ are: the masses $M_{\tilde{L} i j}$ for the slepton $\mathrm{SU}(2)$ doublets $\left(\tilde{\nu}_{L i} \tilde{l}_{L i}\right)$, the masses $M_{\tilde{e} i j}$ for the slepton $\mathrm{SU}(2)$ singlets $\left(\tilde{l}_{R i}\right)$, and the trilinear couplings $A_{i j}$. Our analysis however would only explore the effects of non-diagonal holomorphic or non-holomorphic trilinear couplings that induce mixing in the slepton mass square matrices $\left(M_{\tilde{\ell} L R}^{2}\right){ }^{4}$ Regarding sneutrinos, we may write down a corresponding $3 \times 3$ mass matrix, with respect to the $\left(\tilde{\nu}_{e L}, \tilde{\nu}_{\mu L}, \tilde{\nu}_{\tau L}\right)$ electroweak interaction basis in the sneutrino sector, and we have

$$
\mathcal{M}_{\tilde{\nu}}^{2}=\left(M_{\tilde{\nu} L L}^{2}\right)
$$

where

$$
M_{\tilde{\nu} L L i j}^{2}=M_{\tilde{L} i j}^{2}+\left(\frac{1}{2} M_{Z}^{2} \cos 2 \beta\right) \delta_{i j}
$$

In the above, due to $\mathrm{SU}(2)_{L}$ gauge invariance, the same soft mass $M_{\tilde{L} i j}$ occurs in both the slepton and sneutrino $L L$ mass matrices.

As is known, within the MSSM, LFV decays get no tree-level contribution, just like other FCNC decays. They obtain leading order contributions at loop level via mediation of sleptons (sneutrino)-neutralinos (charginos). Here the source of lepton flavor violation can be from any one (or all) entries- $M_{\tilde{l} L L}^{2}, M_{\tilde{l} L R}^{2}, M_{\tilde{l} R R}^{2}$ of eq. (2.4). But, we will focus on studying the impacts of the non-holomorphic trilinear couplings on the cLFV observables in the NH-MSSM (which would henceforth be called NHSSM), specially in comparison

\footnotetext{
${ }^{3}$ Flavor mixing through trilinear couplings may be generated radiatively in presence of right handed neutrinos (see e.g., [36-38, 54]).

${ }^{4}$ We are taking the off-diagonal soft mass-squared matrix elements in $M_{\tilde{\ell} L L}^{2} \& M_{\tilde{\ell} R R}^{2}$ to be zero at the input scale to probe the effect of trilinear parameters with more clarity. Still, RGE running would generate non-vanishing off-diagonal mass-square matrix elements at a lower scale.
} 
to their holomorphic counterparts. Thus the only source of lepton flavor violation which we would consider here is associated with the left-right slepton mixing. This means that sneutrino-chargino loops will hardly carry any importance in our analysis.

$\boldsymbol{l}_{\boldsymbol{j}} \rightarrow \boldsymbol{l}_{\boldsymbol{i}} \boldsymbol{\gamma}$. Supersymmetric contributions to lepton flavor violating decays $l_{j} \rightarrow l_{i} \gamma$ can be sizable and potentially quite large compared to the same for various other BSM physics models.

The slepton-neutralino and sneutrino-chargino loops mostly contribute to the amplitude of $l_{j} \rightarrow l_{i} \gamma$, through charged particles appearing in the loops. The general amplitude can be written as [36-38],

$$
i \mathcal{M}=i e \epsilon^{\mu *} \bar{u}_{i}(p-q)\left[q^{2} \gamma_{\mu}\left(A_{1}^{L} P_{L}+A_{1}^{R} P_{R}\right)+m_{l_{j}} i \sigma_{\mu \nu} q^{\nu}\left(A_{2}^{L} P_{L}+A_{2}^{R} P_{R}\right)\right] u_{j}(p),
$$

where $\epsilon^{*}$ is the photon polarization vector and $q$ being its momentum. If the photon is on-shell, the first part of the off-shell amplitude vanishes. Thus, we only need to focus on $A_{2}^{L} \& A_{2}^{R}$. The coefficients $A_{2}^{L, R}$ that consist of chargino and neutralino contributions are as given below,

$$
A_{2}^{L, R}=A_{2}^{\left(\tilde{\chi}^{0}\right) L, R}+A_{2}^{\left(\tilde{\chi}^{ \pm}\right) L, R} .
$$

For the case of our current interest, only the NH trilinear couplings may change the slepton mass matrices. So, the flavor violating effects would directly enter from the slepton mass matrix elements into the elements of the diagonalizing matrices. The $\tilde{\chi}^{ \pm}-\tilde{\nu}$ loops are hardly of any importance here because $\mathrm{NH}$ couplings do not affect the sneutrino mass matrix. So $A_{2}^{L}\left(\tilde{\chi}^{0}\right)$ which corresponds to the contribution from real photon emission, is given by [36-38],

$$
A_{2}^{\left(\tilde{\chi}^{0}\right) L}=\frac{1}{32 \pi^{2}} \sum_{A=1}^{4} \sum_{X=1}^{6} \frac{1}{M_{\tilde{l}_{X}}^{2}}\left[N_{i A X}^{L} N_{j A X}^{L *} \frac{1}{12} F_{1}^{N}\left(x_{A X}\right)+N_{i A X}^{L} N_{j A X}^{R *} \frac{m_{\tilde{\chi}_{A}}^{0}}{3 m_{l_{j}}} F_{2}^{N}\left(x_{A X}\right)\right],
$$

where, $x_{A X}=\frac{m_{\chi_{A}^{0}}^{2}}{M_{l_{X}^{2}}^{2}}$. One obtains $A^{R}$ by simply interchanging $L \leftrightarrow R$. The loop functions denoted by $F_{1}, F_{2}$ and the couplings $N_{A X}^{L, R}$ can be read from [36-38, 85].

$$
\begin{aligned}
F_{1}^{N}(x) & =\frac{2}{(1-x)^{4}}\left[1-6 x+3 x^{2}-6 x^{2} \log x\right], \\
\& F_{2}^{N}(x) & =\frac{3}{(1-x)^{3}}\left[1-x^{2}+2 x \log x\right],
\end{aligned}
$$

and

$$
\begin{aligned}
& N_{i A X}^{L}=-\sqrt{2} g_{1}\left(Z_{L}^{i+3, X}\right)^{*} Z_{N}^{1 A}+Y_{l_{i}}\left(Z_{L}^{i, X}\right)^{*} Z_{N}^{3 A}, \\
& N_{i A X}^{R}=\frac{\left(Z_{L}^{i, X}\right)^{*}}{\sqrt{2}}\left(g_{1}\left(Z_{N}^{1 A}\right)^{*}+g_{2}\left(Z_{N}^{2 A}\right)^{*}\right)+Y_{l_{i}}\left(Z_{N}^{3 A}\right)^{*}\left(Z_{L}^{i+3, X}\right)^{*} .
\end{aligned}
$$

Clearly, the couplings $N_{A X}^{L}$ involve $Z_{N} \& Z_{L}$ the neutralino and slepton mixing matrices respectively that transform them from the electroweak basis to the mass basis. $Z_{L}$ 


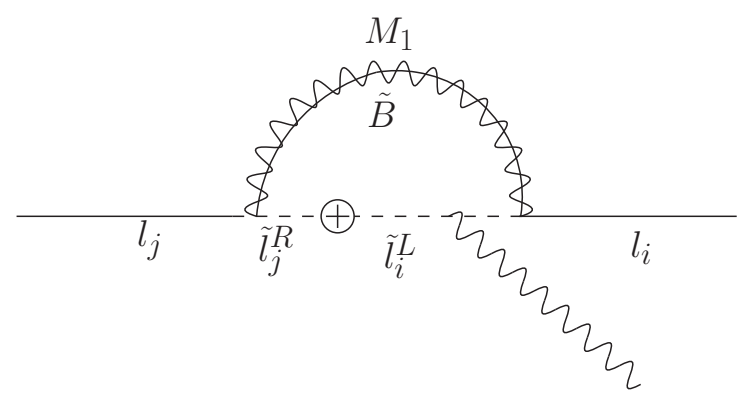

Figure 1. Slepton and neutralino induced Feynman diagram for the process $l_{j} \rightarrow l_{i} \gamma$.

is the $6 \times 6$ slepton mixing matrix that allows for flavor changes in the loop, leading to the flavor violation. One may also evaluate the process using the mass insertions in the slepton mixing matrix (through eq. (2.7)) which depend on the diagonal and non-diagonal entries of the NH trilinear coupling matrix $A_{\ell}^{\left({ }^{\prime}\right)}$. The associated Feynmann diagram are shown in figure 1. For example, in case of $\mu \rightarrow e \gamma$, in the slepton mixing would be induced by $A_{e \mu}-A_{e \mu}^{\prime} \tan \beta$. This indicates a typical domination of $A_{e \mu}^{\prime}$ unless $A_{e \mu}$ is too large or there is much cancellation. Finally, the decay rate is given by,

$$
\Gamma\left(l_{j} \rightarrow l_{i} \gamma\right)=\frac{e^{2}}{16 \pi} m_{l_{j}}^{5}\left(\left|A_{2}^{L}\right|^{2}+\left|A_{2}^{R}\right|^{2}\right) .
$$

$\boldsymbol{l}_{\boldsymbol{j}} \rightarrow \mathbf{3}_{\boldsymbol{i}}$. In the Standard Model (SM), $l_{j} \rightarrow 3 l_{i}$ has a vanishingly small branching fraction, e.g. $\operatorname{Br}(\tau \rightarrow 3 \mu)<10^{-14}$ [86], while various models of beyond the SM may predict this particular process to be of the order of $10^{-10}-10^{-8}$. The current experimental limit of the same BR is of the order of few times $10^{-8}$ [87-90] which has much better sensitivity compared to the 3 -body decays of $\mu$. The main experimental obstacle to improve the sensitivity with $\tau$ leptons is the fact that $\tau$ is not produced in large numbers. The amplitude for $l_{j} \rightarrow 3 l_{i}$ comprises of contributions from $\gamma, Z, \phi(=h, H, A)$ penguin diagrams and the box diagram as shown in figure 2 with slepton (sneutrino)-neutralino (chargino) appearing inside the loop. Detailed expressions for the diagrams may be found in [36-38, 42, 53]. The effects of NH off-diagonal elements toward LFV appear via slepton mass matrices. This induces an effective vertex $\phi, \gamma, Z-l_{i}-\bar{l}_{j}$ which in turn leads to processes like $l_{j} \rightarrow 3 l_{i}$. All the penguin processes would further be boosted in case of non-holomorphic couplings via additional $\tan \beta$ factor (see eq. (2.7)). Similar to the MSSM case the dominance of $\gamma$-penguins in the cLFV processes in NHSSM also holds good. This is irrespective of the fact that Higgs penguin contributions that scale as $\tan ^{6} \beta$ are expected to be large for large $\tan \beta[42,91]$. The dominance of $\gamma$-penguins is also valid in relation to the $\mathrm{Z}$ and box contributions. ${ }^{5}$

$\phi(\boldsymbol{h}, \boldsymbol{H}, \boldsymbol{A}) \rightarrow \boldsymbol{l}_{\boldsymbol{i}} \overline{\boldsymbol{l}}_{\boldsymbol{j}}$. Flavor changing Higgs decays can play significant roles for investigating lepton flavor violation. The same Higgs mediated penguin diagrams, induced by $\phi-\tilde{l}_{i}-\tilde{\tilde{l}}_{j}$ vertex, may effectively contribute in $\phi-l_{i}-\bar{l}_{j}$ vertex through loops leading to Higgs flavor violating decays. The effective Lagrangian representing the interaction

\footnotetext{
${ }^{5}$ For relative contributions see figure 5 of ref. [53].
} 


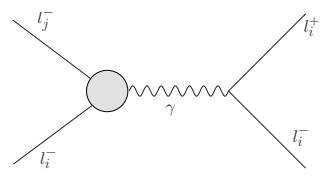

(a)

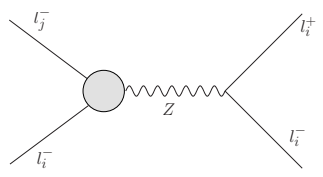

(b)

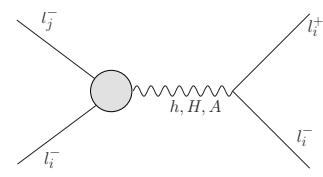

(c)

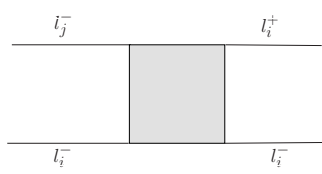

(d)

Figure 2. Photon, Z-boson and neutral Higgs boson mediated penguin diagrams and box-type of diagram contributing to $l_{j} \rightarrow 3 l_{i}$ decay.
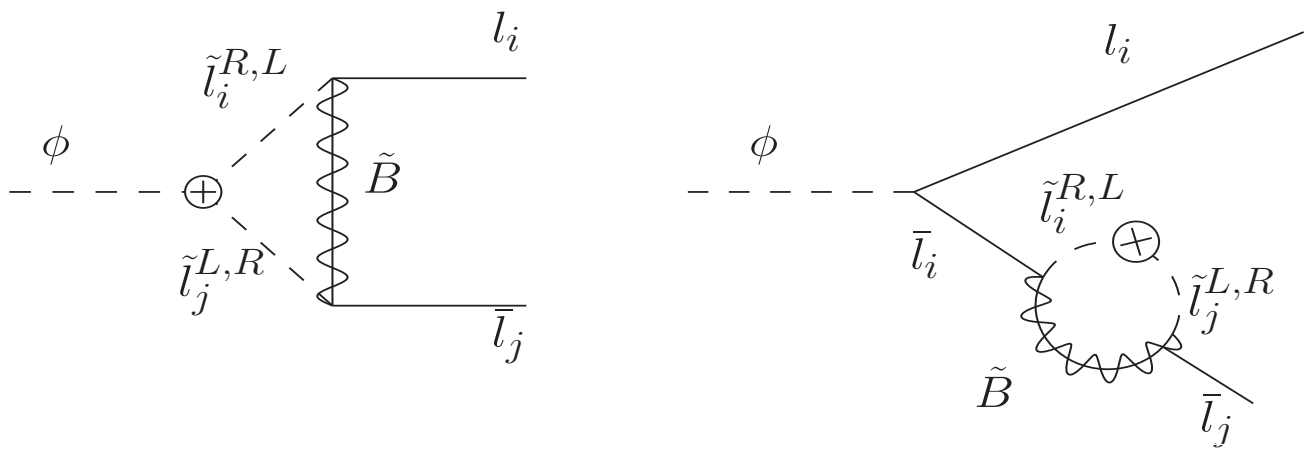

Figure 3. One loop diagrams contributing to computation of $\operatorname{Br}\left(\phi \rightarrow l_{i} \overline{l_{j}}\right)$ with LR \& RL mixing.

between neutral Higgs boson and charged leptons is given by [42, 91],

$$
-\mathcal{L}_{\text {eff }}=\bar{e}_{R}^{i} y_{e_{i i}}\left[\delta_{i j} H_{d}^{0}+\left(\epsilon_{1} \delta_{i j}\left(A_{i j} H_{d}^{0}-\left(\mu+A_{i j}^{\prime}\right) H_{u}^{0 *}\right)\right)+\epsilon_{2 i j}\left(A_{i j} H_{d}^{0}-A_{i j}^{\prime} H_{u}^{0 *}\right)\right] l_{L}^{j}+\text { h.c. }
$$

The first term of the above equation denotes the Yukawa interaction whereas $\epsilon_{1}$ encodes the corrections to the charged lepton Yukawa couplings from flavor conserving loops [42]. The last term in eq. (2.17) corresponds to the source of flavor violation through the insertion of $\left(A_{i j}-A_{i j}^{\prime} \tan \beta\right)$ in the slepton arms inside the loops. $\epsilon_{2}$ arises out of loop functions involving neutralino and slepton masses owing to various cLFV processes. The effective Lagrangian in eq. (2.17) esentially generates all the off-diagonal Yukawa couplings radiatively if the respective holomorphic (non-holomorphic) trilinear couplings $A_{i j}\left(A_{i j}^{\prime}\right)$ are non zero. This in turn produces flavor violating decays of Higgs scalars or lepton 3-body decays induced by the Higgs penguins. Among the Higgs mediated diagrams, typically dominant contributions come from the CP-odd Higgs exchange $A$ for large $\tan \beta$. This may be understood from the effective Lagrangian describing couplings of the physical Higgs bosons to the leptons, which can be derived from eq. (2.17) [42, 91].

$$
-\mathcal{L}_{i \neq j}^{\mathrm{eff}}=\left(2 G_{F}^{2}\right)^{1 / 4} \frac{m_{E_{i}} \kappa_{i j}^{E}}{\cos \beta}\left(\bar{e}_{R}^{i} l_{L}^{j}\right)[\cos (\alpha-\beta) h+\sin (\alpha-\beta) H-i A]+\text { h.c. }
$$

Here, $\alpha$ is the CP-even Higgs mixing angle and $\tan \beta=v_{u} / v_{d}$, and

$$
\kappa_{i j}^{E}=\frac{\epsilon_{2}\left(A_{i j}-A_{i j}^{\prime} \tan \beta\right)}{\left.\left[1+\left(\epsilon_{1}\left(A_{i i}-\left(\mu+A_{i i}^{\prime}\right) \tan \beta\right)\right)+\epsilon_{2}\left(A_{i i}-A_{i i}^{\prime} \tan \beta\right)\right)\right]}
$$

Since the cLFV branching ratios are proportional to $\left(\kappa_{i j}^{E}\right)^{2}$, from the above equation it is clear that the non-holomorphic trilinear couplings via $\tan \beta$ enhancement may have 
greater importance towards Higgs mediated processes. In fact, all the Higgs mediated flavor violating observables may receive large boost, while assuming no cancellation in eq. (2.19) or if holomorphic $A_{i j}$ is negligible compared to $A_{i j}^{\prime}$. In case of flavor violating Higgs decays the branching fraction $\phi_{k} \rightarrow \mu \tau$ where Higgs bosons $h, H, A$ are denoted as $\phi_{k}$ for $k=1,2,3$ can be related to the flavor conserving decay $\phi_{k} \rightarrow \tau \tau$ as follows [49].

$$
\operatorname{Br}\left(\phi_{k} \rightarrow \mu \tau\right)=\tan ^{2} \beta\left(\left|\kappa_{\tau \mu}^{E}\right|^{2}\right) C_{\Phi} \operatorname{Br}\left(\phi_{k} \rightarrow \tau \tau\right),
$$

where we used $1 / \cos ^{2} \beta \simeq \tan ^{2} \beta$. The coefficients $C_{\Phi}$ are given by,

$$
C_{h}=\left[\frac{\cos (\beta-\alpha)}{\sin \alpha}\right]^{2}, \quad C_{H}=\left[\frac{\sin (\beta-\alpha)}{\cos \alpha}\right]^{2}, \quad C_{A}=1 .
$$

\section{Analysis of charge breaking minima}

Absence of any flavor changing neutral current (FCNC) significantly constrains the offdiagonal elements in the mass and trilinear coupling matrices. However, the Charge and Color Breaking(CCB) constraints are more robust than the corresponding FCNC data [93]. In a multi-scalar theory, the existence of several vacua and choice of the desired electroweak symmetry breaking put strong constraints on the allowed parameter space. In this context, we first put the effort to analyze the charge breaking bounds for two generations of sleptons associated with the $(\tilde{\mu}-\tilde{\tau})$ sector. Here trilinear couplings can accommodate the offdiagonal entries of both the holomorphic and non-holomorphic soft SUSY breaking terms. Then we will generalize it for all the three generations of sleptons.

Three basic components of tree level scalar potential $V_{0}$ are the F-term, D-term and the soft breaking terms, $V_{0}=V_{F}+V_{D}+V_{\text {soft }}$. The constituents of $V_{0}$ are given as follows,

$$
V_{F}=\sum_{a}\left|\frac{\partial W}{\partial \phi_{a}}\right|^{2}
$$

here the superpotential $\mathrm{W}$ is given by eq. (2.1). The D-term part gives additional quartic terms for scalar potential associated with gauge couplings $g_{a}$.

$$
V_{D}=\frac{1}{2} \sum_{a} g_{a}^{2}\left(\sum_{a} \phi_{a}^{\dagger} T^{a} \phi_{a}\right)^{2}
$$

The Holomorphic and non-holomorphic soft terms in $\mathrm{V}$ (and hence in $-\mathcal{L}$ ) can be written as,

$$
\begin{aligned}
V_{\mathrm{soft}} & =\sum M_{\phi_{a}}^{2}\left|\phi_{a}\right|^{2}+\left(\tilde{u}_{i R}^{*} \mathbf{A}_{\mathbf{u} i j} \tilde{q}_{j L} \cdot H_{u}+\tilde{d}_{i R}^{*} \mathbf{A}_{\mathbf{d} i j} \tilde{q}_{j L} \cdot H_{d}+\tilde{e}_{i R}^{*} \mathbf{A}_{\mathbf{e} i j} \tilde{\ell}_{j L} \cdot H_{d}+\text { h.c. }\right), \\
V_{\mathrm{soft}}^{N H} & =\tilde{u}_{i R}^{*} \mathbf{A}_{\mathbf{u} i j}^{\prime} \tilde{q}_{j L} \cdot H_{d}^{*}+\tilde{d}_{i R}^{*} \mathbf{A}_{\mathbf{d} i j}^{\prime} \tilde{q}_{j L} \cdot H_{u}^{*}+\tilde{e}_{i R}^{*} \mathbf{A}_{\mathbf{e} i j}^{\prime} \tilde{\ell}_{j L} \cdot H_{u}^{*}+h . c .
\end{aligned}
$$

In the above, $\phi_{a}$ runs over all the scalar components of chiral superfields. The full MSSM scalar potential may indeed have several minima where squarks or sleptons may additionally acquire non-zero vevs which may in turn lead to charge and/or color breaking vacua. Since the violation of charge and/or color quantum number is yet to be observed, it is understood that the universe at present is at a ground state which is Standard 
Model like (SML) [102], with only neutral components of the Higgs scalars acquiring vevs. A priori, it indicates that those parts of the multi-dimensional parameter space corresponding to MSSM scalar potential that allow a deeper charge and color breaking (CCB) minima [94-100, 102] should be excluded. The dangerous directions could be associated with unacceptably large trilinear couplings, in particular $A_{t}, A_{b}$, the ones associated with top and bottom Yukawa couplings. Thus, one may have a CCB vacuum that is deeper than the desired EWSB vacuum. Analyses of CCB constraints in MSSM may be seen in refs. [93, 95, 101-103] a related study on non-holomorphic soft terms was made in ref. [104]. Here one should note that the rate of tunneling from SML false vacuum to such CCB true vacuum is roughly proportional to $e^{-a / y^{2}}$, where $a$ is a constant of suitable dimension that can be determined via field theoretic calculations and $y$ is the Yukawa coupling. The tunneling rate is enhanced for large Yukawa couplings [105-110], thus leading to large effects from the third generation of sfermions.

But it is not always true that, trilinear terms of third generations of squarks/sleptons are the most important for charge and color breaking minima. With the variation of nonholomorphic soft terms, there can be significant changes in all of the corresponding Yukawa couplings through loops $[111]^{6}$ and other two generations of squarks/sleptons can have notable effect in charge and color breaking condition [104]. Here we will study the analytic expressions for only charge breaking minima for slepton soft masses and slepton trilinear couplings (both holomorphic and non-holomorphic) considering all the three generations. We will particularly generalize our studies to include first the effect of non-vanishing, nondiagonal trilinear soft terms. Furthermore, we will consider only the case of absolute stability of the vacuum, i.e. without trying to analyze any tunneling effect.

\subsection{Charge breaking with flavor violation in MSSM}

In this subsection we first analyze the effect of non-vanishing off-diagonal entries $A_{i j}^{\left({ }^{\prime}\right)}$ on charge breaking in MSSM. The relevant terms in $V_{F}, V_{D}$ and $V_{\text {soft }}$ of MSSM related to the slepton sector are as given below,

$$
\begin{aligned}
V_{F}= & \left|\mu^{*} H_{u}^{-}-\tilde{\nu}_{i L}^{*} y_{i j} \tilde{e}_{j R}\right|^{2}+\left|\mu^{*} H_{u}^{0}-\tilde{e}_{i L}^{*} y_{i j} \tilde{e}_{j R}\right|^{2} \\
& +\sum_{l}\left|y_{i j} H_{d}^{0} \tilde{l}_{j L}\right|^{2}+y_{i j} y_{i j^{\prime}}^{\star} \tilde{e}_{j R}^{*} \tilde{e}_{j^{\prime} R}\left(\left|H_{d}^{0}\right|^{2}+H_{d}^{+} H_{d}^{-}\right), \\
V_{D}= & V_{D_{Y}}+V_{\vec{D}} \\
= & \frac{g_{1}^{2}}{8}\left(\left|H_{u}^{0}\right|^{2}-\left|H_{d}^{0}\right|^{2}-\left|\tilde{l}_{i L}\right|^{2}+2\left|\tilde{e}_{i R}\right|^{2}\right)^{2}+\frac{g_{2}^{2}}{8}\left(\left|H_{u}^{0}\right|^{2}-\left|H_{d}^{0}\right|^{2}+\left|\tilde{l}_{i L}\right|^{2}\right)^{2}, \\
V_{\text {soft }}^{l}= & \tilde{l}_{i L}^{\dagger}\left(M_{\tilde{L}}^{2}\right)_{i j} \tilde{l}_{j L}+\tilde{e}_{i R}^{*}\left(M_{\tilde{e}}^{2}\right)_{i j} \tilde{e}_{j R}^{\dagger}+\left[\tilde{e}_{i R}^{*} \mathbf{A}_{\mathrm{lij}} \tilde{l}_{j L} \cdot H_{d}+h . c\right], \\
V_{\text {soft }}^{H}= & m_{H_{d}}^{2}\left|H_{d}^{0}\right|^{2}+m_{H_{u}}^{2}\left|H_{u}^{0}\right|^{2}-2 R e\left(B \mu H_{d}^{0} H_{u}^{0}\right) .
\end{aligned}
$$

\footnotetext{
${ }^{6}$ In this context we note that the radiative effects will be larger in the strongly interacting sector as in the analysis of [111] involving $Y_{b}$. In the context of the present analysis, both the relevant diagonal and off-diagonal Yukawa couplings involving $\tau$ may get affected. However, a change in the off-diagonal Yukawas will be manifestly more relevant in eq. (2.19) via the appearance of $\epsilon_{2}$ in the numerator. In contrast to the above, the denominator can hardly be different from unity unless the diagonal NH trilinear soft parameters are too large.
} 
Below, we collect the terms, originating from $V_{F}, V_{D}, V_{\text {soft }}^{\tilde{l}}$ and $V_{\text {soft }}^{H}$ appearing in the diagonal and off-diagonal elements for the 2 nd and 3rd generations of sleptons (viz. smuon and stau).

$$
\begin{aligned}
V_{\mu}^{\text {diag }}= & \tilde{\mu}_{L}^{*}\left(M_{\tilde{L}_{22}}^{2}+\left|y_{\mu} H_{d}^{0}\right|^{2}\right) \tilde{\mu}_{L}+\tilde{\mu}_{R}^{*}\left(M_{\tilde{e}_{22}}^{2}+\left|y_{\mu} H_{d}^{0}\right|^{2}\right) \tilde{\mu}_{R} \\
& +\left[\tilde{\mu}_{L}^{*}\left(A_{\mu} h_{d}-\mu^{*} y_{\mu} h_{u}\right) \tilde{\mu}_{R}+h . c\right]+\left|y_{\mu}\right|^{2}\left|\tilde{\mu}_{L}\right|^{2}\left|\tilde{\mu}_{R}\right|^{2}, \\
V_{\tau}^{\text {diag }}= & \tilde{\tau}_{L}^{*}\left(M_{\tilde{L}_{33}}^{2}+\left|y_{\tau} H_{d}^{0}\right|^{2}\right) \tilde{\tau}_{L}+\tilde{\tau}_{R}^{*}\left(M_{\tilde{e}_{33}}^{2}+\left|y_{\tau} H_{d}^{0}\right|^{2}\right) \tilde{\tau}_{R} \\
& +\left[\tilde{\tau}_{L}^{*}\left(A_{\tau} H_{d}^{0}-\mu^{*} y_{\tau} H_{u}^{0}\right) \tilde{\tau}_{R}+h . c\right]+\left|y_{\tau}\right|^{2}\left|\tilde{\tau}_{L}\right|^{2}\left|\tilde{\tau}_{R}\right|^{2}, \\
V_{\mu \tau}= & \tilde{\mu}_{L}^{*} M_{\tilde{L}_{23}}^{2} \tilde{\tau}_{L}+\tilde{\mu}_{R}^{*}\left[M_{\tilde{e}_{23}}^{2}+\left|\mathbf{y}_{\mu \tau} H_{d}^{0}\right|^{2}\right] \tilde{\tau}_{R}+\left[\tilde{\mu}_{L}^{*}\left(A_{\mu \tau} H_{d}^{0}-\mu^{*} y_{\mu \tau} H_{u}^{0}\right) \tilde{\tau}_{R}+h . c\right] \\
& +\left|y_{\mu \tau}\right|^{2}\left|\tilde{\mu}_{L}\right|^{2}\left|\tilde{\tau}_{R}\right|^{2}+\tilde{\tau}_{L}^{*} M_{\tilde{e}_{32}}^{2} \tilde{\mu}_{L}+\tilde{\tau}_{R}^{*}\left[M_{\tilde{e}_{32}}^{2}+\left|\mathbf{y}_{\tau \mu} H_{d}^{0}\right|^{2}\right] \tilde{\mu}_{R} \\
& +\left[\tilde{\tau}_{L}^{*}\left(A_{\tau \mu} H_{d}^{0}-\mu^{*} y_{\tau \mu} H_{u}^{0}\right) \tilde{\mu}_{R}+h . c\right]+\left|y_{\tau \mu}\right|^{2}\left|\tilde{\tau}_{L}\right|^{2}\left|\tilde{\mu}_{R}\right|^{2}, \\
V_{H}= & \left(m_{H_{u}}^{2}+|\mu|^{2}\right)\left|H_{u}^{0}\right|^{2}+\left(m_{H_{d}}^{2}+|\mu|^{2}\right)\left|H_{d}^{0}\right|^{2}, \\
V_{D}= & \frac{g_{1}^{2}}{8}\left(\left|H_{u}^{0}\right|^{2}-\left|H_{d}^{0}\right|^{2}-\left|\tilde{\mu}_{L}\right|^{2}-\left|\tilde{\tau}_{L}\right|^{2}+2\left|\tilde{\mu}_{R}\right|^{2}+2\left|\tilde{\tau}_{R}\right|^{2}\right)^{2} \\
& +\frac{g_{2}^{2}}{8}\left(\left|H_{u}^{0}\right|^{2}-\left|H_{d}^{0}\right|^{2}+\left|\tilde{\mu}_{L}\right|^{2}+\left|\tilde{\tau}_{L}\right|^{2}\right)^{2} .
\end{aligned}
$$

In the first place we consider non-vanishing vevs for the neutral components of the two Higgs scalars and the stau and smuon fields. The latter are responsible for the generation of charge breaking minima. Allowing both $H_{u}^{0}$ and $H_{d}^{0}$ to fluctuate in the positive and negative directions, we choose to constrain the slepton fields with a particular scalar field value $\phi$. In this specific direction one has,

$$
\begin{aligned}
\left|\tilde{\tau_{L}}\right|=\left|\tilde{\tau_{R}}\right| & =\alpha \phi, \\
\left|\tilde{\mu_{L}}\right|=\left|\tilde{\mu_{R}}\right| & =\beta \phi, \\
H_{d}^{0} & =\phi, \\
H_{u}^{0} & =\eta \phi .
\end{aligned}
$$

with $\eta$ being any real number and $\alpha, \beta$ to be real and positive. The total tree-level scalar potential involving Higgs, smuon and stau fields, assuming $\mu$ to be real and $y_{i j}$ or $A_{i j}$ referring to real symmetric matrices, reduces to,

$$
V_{\tilde{l}, H}=A \phi^{2}+B \phi^{3}+C \phi^{4},
$$

where,

$$
\begin{aligned}
A= & \alpha^{2}\left(M_{\tilde{L}_{33}}^{2}+M_{\tilde{e}_{33}}^{2}\right)+\beta^{2}\left(M_{\tilde{L}_{22}}^{2}+M_{\tilde{e}_{22}}^{2}\right)+2 \alpha \beta\left(M_{\tilde{L}_{23}}^{2}+M_{\tilde{e}_{23}}^{2}\right)+m_{H_{d}}^{2}+\eta^{2} m_{H_{u}}^{2} \\
& +\left(1+\eta^{2}\right)|\mu|^{2}-2 B \mu \eta, \\
B= & 2 \alpha^{2}\left(A_{\tau}-\mu y_{\tau} \eta\right)+2 \beta^{2}\left(A_{\mu}-\mu y_{\mu} \eta\right)+4 \alpha \beta\left(A_{\mu \tau}-\mu y_{\mu \tau} \eta\right), \\
C= & \frac{g_{1}^{2}+g_{2}^{2}}{8}\left(\eta^{2}-1+\beta^{2}+\alpha^{2}\right)^{2}+\left(2+\alpha^{2}\right) \alpha^{2} y_{\tau}^{2}+\left(2+\beta^{2}\right) \beta^{2} y_{\mu}^{2}+2 \alpha^{2} \beta^{2} y_{\mu \tau}^{2} .
\end{aligned}
$$

We require that the minima at $\langle\phi\rangle=0$ should be deeper than a minima with $\langle\phi\rangle \neq 0$ and this is possible when $B^{2}(\alpha, \beta, \eta)<4 A(\alpha, \beta, \eta) C(\alpha, \beta, \eta)$. Here we consider a scenario 
with 6 vevs corresponding to $\mathrm{L}$ and $\mathrm{R}$ components of smuon and stau fields apart from the neutral Higgs fields corresponding to eq. (3.5).

We want to have the most stringent condition that would avoid the charge breaking minima. Thus, in the D-flat direction, which explicitly demands that all the $g_{i}^{2}$ terms in the tree level scalar potential to be absent, we choose,

$$
\alpha=\frac{1}{\sqrt{2}}, \quad \beta=\frac{1}{\sqrt{2}}, \quad \eta=0,
$$

so that, $\left(\eta^{2}-1+\alpha^{2}+\beta^{2}\right)=0$.

Thus we obtain,

$$
\begin{aligned}
A & =\frac{1}{2}\left(M_{\tilde{L}_{33}}^{2}+M_{\tilde{e}_{33}}^{2}\right)+\frac{1}{2}\left(M_{\tilde{L}_{22}}^{2}+M_{\tilde{e}_{22}}^{2}\right)+\left(M_{\tilde{L}_{23}}^{2}+M_{\tilde{e}_{23}}^{2}\right)+m_{H_{d}}^{2}+|\mu|^{2}, \\
B & =A_{\tau}+A_{\mu}+2 A_{\mu \tau} \\
C & =\frac{5}{4}\left(y_{\tau}^{2}+y_{\mu}^{2}+\frac{2}{5} y_{\mu \tau}^{2}\right) .
\end{aligned}
$$

With $B^{2}(\alpha, \beta, \eta)<4 A(\alpha, \beta, \eta) C(\alpha, \beta, \eta)$ one obtains the following that would avoid a charge breaking minima

$$
\begin{aligned}
\left(A_{\tau}+A_{\mu}+2 A_{\mu \tau}\right)^{2}< & 5\left(y_{\tau}^{2}+y_{\mu}^{2}+\frac{2}{5} y_{\mu \tau}^{2}\right) \\
& \times\left[\frac{1}{2}\left(M_{\tilde{L}_{33}}^{2}+M_{\tilde{e}_{33}}^{2}\right)+\frac{1}{2}\left(M_{\tilde{L}_{22}}^{2}+M_{\tilde{e}_{22}}^{2}\right)+\left(M_{\tilde{L}_{23}}^{2}+M_{\tilde{e}_{23}}^{2}\right)+m_{H_{d}}^{2}+|\mu|^{2}\right] .
\end{aligned}
$$

Including all the three generations of leptons, eq. (3.7) generalizes into the following.

$$
\begin{aligned}
\left(\sum_{e, \mu, \tau} A_{i}+2 \sum_{i \neq j} A_{i j}\right)^{2}< & 5\left(\sum_{e, \mu, \tau} y_{i}^{2}+\frac{2}{5} \sum_{i \neq j} y_{i j}^{2}\right) \\
& \times\left[\frac{1}{2} \sum_{e, \mu, \tau}\left(M_{\tilde{L}_{i i}}^{2}+M_{\tilde{e}_{i i}}^{2}\right)+\sum_{i \neq j}\left(M_{\tilde{L}_{i j}}^{2}+M_{\tilde{e}_{i j}}^{2}\right)+m_{H_{d}}^{2}+|\mu|^{2}\right] .
\end{aligned}
$$

\subsection{Charge breaking condition in NHSSM}

With non-holomorphic terms in $V_{\text {soft }}$ involving only the appropriate trilinear $\mathrm{NH}$ couplings we will have following extra terms,

$$
V_{N H}^{\tilde{l}}=-\left[\tilde{\mu}_{L}^{*}\left(A_{\mu}^{\prime} H_{u}^{0}\right) \tilde{\mu}_{R}+\tilde{\tau}_{L}^{*}\left(A_{\tau}^{\prime} H_{u}^{0}\right) \tilde{\tau}_{R}+\tilde{\mu}_{L}^{*}\left(A_{\mu \tau}^{\prime} H_{u}^{0}\right) \tilde{\tau}_{R}+\tilde{\tau}_{L}^{*}\left(A_{\tau \mu}^{\prime} H_{u}^{0}\right) \tilde{\mu}_{R}+\text { h.c }\right] .
$$

Considering the direction mentioned in (3.5), we find the following for NHSSM.

$$
\begin{aligned}
V_{\tilde{l}, H}= & \left\{\alpha^{2}\left(M_{L_{33}}^{2}+M_{e_{33}}^{2}\right)+\beta^{2}\left(M_{L_{22}}^{2}+M_{e_{22}}^{2}\right)+2 \alpha \beta\left(M_{L_{23}}^{2}+M_{e_{23}}^{2}\right)\right. \\
& \left.+m_{H_{d}}^{2}+\eta^{2} m_{H_{u}}^{2}+\left(1+\eta^{2}\right)|\mu|^{2}-2 B \mu \eta\right\} \phi^{2} \\
& +\left\{2 \alpha^{2}\left(A_{\tau}-A_{\tau}^{\prime} \eta-\mu y_{\tau} \eta\right)+2 \beta^{2}\left(A_{\mu}-A_{\mu}^{\prime} \eta-\mu y_{\mu} \eta\right)+4 \alpha \beta\left(A_{\mu \tau}-A_{\mu \tau}^{\prime} \eta-\mu y_{\mu \tau} \eta\right)\right\} \phi^{3} \\
& +\left\{\frac{g_{1}^{2}+g_{2}^{2}}{8}\left(\eta^{2}-1+\beta^{2}+\alpha^{2}\right)^{2}+\left(2+\alpha^{2}\right) \alpha^{2} y_{\tau}^{2}+\left(2+\beta^{2}\right) \beta^{2} y_{\mu}^{2}+2 \alpha^{2} \beta^{2} y_{\mu \tau}^{2}\right\} \phi^{4} .
\end{aligned}
$$


Earlier, we obtained the most stringent bound along D-flat direction which requires $\eta=0$. But, the same choice is insufficient to provide any bound on the NH trilinear couplings. This is simply because $\eta$ gets multiplied with the $\mathrm{NH}$ trilinear parameters in eq. (3.9). Instead we assume $\alpha=\frac{1}{\sqrt{2}}, \beta=\frac{1}{\sqrt{2}}, \eta=1$, which leads to a stringent bound on the $A_{\mu}^{\prime}$ and $A_{\tau}^{\prime}$ for avoiding a deeper charge breaking minima in NHSSM. The bound can be read as shown below:

$$
\begin{aligned}
& {\left[A_{\tau}-\left(\mu y_{\tau}+A_{\tau}^{\prime}\right)+A_{\mu}-\left(\mu y_{\mu}+A_{\mu}^{\prime}\right)+2\left\{A_{\mu \tau}-\left(\mu y_{\mu \tau}+A_{\mu \tau}^{\prime}\right)\right\}\right]^{2}} \\
& <4 \cdot \frac{1}{4}\left(\frac{g_{1}^{2}+g_{2}^{2}}{2}+5 y_{\tau}^{2}+5 y_{\mu}^{2}+2 y_{\mu \tau}^{2}\right) \\
& \times\left[\frac{1}{2}\left(M_{\tilde{L}_{33}}^{2}+M_{\tilde{e}_{33}}^{2}\right)+\frac{1}{2}\left(M_{\tilde{L}_{22}}^{2}+M_{\tilde{e}_{22}}^{2}\right)+\left(M_{\tilde{L}_{23}}^{2}+M_{\tilde{e}_{23}}^{2}\right)+m_{H_{u}}^{2}+m_{H_{d}}^{2}+2|\mu|^{2}-2 B \mu\right] .
\end{aligned}
$$

Again dealing with all three generations of sleptons eq. (3.10) becomes,

$$
\begin{aligned}
\left(\sum_{e, \mu, \tau}\right. & \left.\left\{A_{i}-\left(A_{i}^{\prime}+\mu y_{i}\right)\right\}+2 \sum_{i \neq j}\left\{A_{i j}-\left(A_{i j}^{\prime}+\mu y_{i j}\right)\right\}\right)^{2} \\
< & \left(\frac{g_{1}^{2}+g_{2}^{2}}{2}+5 \sum_{e, \mu, \tau} y_{i}^{2}+2 \sum_{i \neq j} y_{i j}^{2}\right) \\
& \times\left[\frac{1}{2} \sum_{e, \mu, \tau}\left(M_{\tilde{L}_{i i}}^{2}+M_{\tilde{e}_{i i}}^{2}\right)+\sum_{i \neq j}\left(M_{\tilde{L}_{i j}}^{2}+M_{\tilde{e}_{i j}}^{2}\right)+m_{H_{u}}^{2}+m_{H_{d}}^{2}+2|\mu|^{2}-2 B \mu\right] .
\end{aligned}
$$

Eq. (3.11) represents the most general condition to avoid a charge breaking minima considering all kinds of soft breaking terms for three generations of fermions. Stringent constraints on individual non-holomorphic diagonal and non-diagonal trilinear couplings may be derived from (3.11). However, rather than following the bounds on the individual couplings, hereafter we will use eq. (3.8) and (3.11) in all our results to constrain the trilinear parameters.

\section{Status of different LFV decays}

Here we would summarize the experimental efforts and the degree of current and future sensitivities of several cLFV processes.

In the radiative decay of $l_{j} \rightarrow l_{i} \gamma$, the experiment leading to the most stringent constraint is MEG [112], which is currently operational at the Paul Scherrer Institute in Switzerland. This searches for the radiative process $\mu \rightarrow e \gamma$. The MEG collaboration proclaimed a new limit on the rate for this process based on the analysis of a data set with $3.6 \times 10^{14}$ stopped muons. The non-observation of the cLFV process leads to $\operatorname{Br}(\mu \rightarrow$ $e \gamma)<4.2 \times 10^{-13}[112]$, which is four times more stringent than the earlier one, obtained by the same collaboration. Moreover, the MEG collaboration has announced plans for future upgrades leading to a sensitivity of about $6 \times 10^{-14}$ after 3 years of data acquisition [113].

The most interesting results in the near future are expected in $\mu \rightarrow 3 e$ and $\mu-e$ conversion in nuclei. The Mu3e experiment $[114,115]$ is designed to search for charged 


\begin{tabular}{|c|c|c|}
\hline LFV Process & Present Bound & Future Sensitivity \\
\hline$B r(\mu \rightarrow e \gamma)$ & $4.2 \times 10^{-13}[112]$ & $6 \times 10^{-14}[113]$ \\
$B r(\tau \rightarrow e \gamma)$ & $3.3 \times 10^{-8}[119]$ & $\sim 3 \times 10^{-9}[121]$ \\
$B r(\tau \rightarrow \mu \gamma)$ & $4.4 \times 10^{-8}[119]$ & $\sim 3 \times 10^{-9}[119]$ \\
$B r(\mu \rightarrow 3 e)$ & $1.0 \times 10^{-12}[116]$ & $10^{-16}[114,117]$ \\
$B r(\tau \rightarrow 3 e)$ & $2.7 \times 10^{-8}[88]$ & $\sim 10^{-9}[121]$ \\
$B r(\tau \rightarrow 3 \mu)$ & $3.3 \times 10^{-8}[88]$ & $\sim 10^{-9}[121]$ \\
$\operatorname{Br}\left(\tau^{-} \rightarrow e^{-} \mu^{+} \mu^{-}\right)$ & $2.7 \times 10^{-8}[88]$ & $\sim 10^{-9}[121]$ \\
$\operatorname{Br}\left(\tau^{-} \rightarrow \mu^{-} e^{+} e^{-}\right)$ & $1.8 \times 10^{-8}[88]$ & $\sim 10^{-9}[121]$ \\
$\operatorname{Br}\left(\tau^{-} \rightarrow e^{+} \mu^{-} \mu^{-}\right)$ & $1.7 \times 10^{-8}[88]$ & $\sim 10^{-9}[121]$ \\
$\operatorname{Br}\left(\tau^{-} \rightarrow \mu^{+} e^{-} e^{-}\right)$ & $1.5 \times 10^{-8}[88]$ & $\sim 10^{-9}[121]$ \\
$B r(\tau \rightarrow \mu \eta)$ & $2.3 \times 10^{-8}[122]$ & $\sim 10^{-10}[123]$ \\
$\operatorname{Br}\left(\tau \rightarrow \mu \eta^{\prime}\right)$ & $3.8 \times 10^{-8}[122]$ & $\sim 10^{-10}[123]$ \\
$\operatorname{Br}\left(\tau \rightarrow \mu \pi^{0}\right)$ & $2.2 \times 10^{-8}[122]$ & $\sim 10^{-10}[123]$ \\
\hline
\end{tabular}

Table 1. Current Experimental situation and future sensitivities for principal LFV processes.

lepton flavor violation in the process $\mu \rightarrow 3 e$ with a branching ratio sensitivity of $10^{-16}$. The present limit on the $\mu \rightarrow 3 e$ has been set by the SINDRUM experiment [116]. As no signal was observed, branching fractions larger than $1.0 \times 10^{-12}$ were excluded at $90 \%$ confidence limit (CL). For the upcoming Mu3e experiment, in phase I, a branching fraction of $5.2 \times 10^{-15}$ can be measured or excluded at 90\% CL [117].

In the recent times, the most actively studied cLFV processes are the rare $\tau$ decays. $\tau$ pairs are abundantly produced at the $B$ factories e.g., in the BELLE [118] \& BABAR [119] collaborations. There are significant improvement on most of the cLFV modes of the $\tau$ decays, though any of them has not been discovered yet. The LHCb collaboration also announced the first ever bounds on $\tau \rightarrow 3 \mu$ in a hadron collider [120]. The current experimental upper limits on the LFV radiative decays $[112,118,119]$ are collected in the table 1 with references.

Apart from the leptonic decays with LFV there are bounds from LFV Higgs decays. The first direct search of LFV Higgs decays were performed by CMS and ATLAS Collaborations [124, 125]. A slight excess of signal events with a significance of $2.4 \sigma$ was observed by CMS at $8 \mathrm{TeV}$ data but, that early peak by CMS is not supported at $13 \mathrm{TeV}$ anymore, finding $\operatorname{Br}(h \rightarrow \mu \tau)<1.20 \%$ with $2.3 \mathrm{fb}^{-1}$ data [126]. Subsequently CMS confirmed the disappearance of that excess $[127,128]$. Additionally, at $13 \mathrm{TeV}$ with integrated luminosity of $35.9 \mathrm{fb}^{-1}$, no significant excess over the Standard Model expectation is observed. The observed (expected) upper limits on the lepton flavor violating branching fractions of the Higgs boson are $\mathrm{Br}(h \rightarrow \mu \tau)<0.28 \%(0.37 \%)$ and $\mathrm{Br}(h \rightarrow e \tau)<0.47 \%(0.34 \%)$ at $95 \%$ confidence level. These results are used to derive upper limits on the off-diagonal $\mu \tau$ and $e \tau$ Yukawa couplings [128]. These limits on the lepton flavor violating branching fractions 


\begin{tabular}{|c|c|}
\hline LFV Higgs decays & Present upper limit \\
\hline$B r(h \rightarrow \mu \tau)$ & $2.5 \times 10^{-3}[127,128]$ \\
$B r(h \rightarrow e \tau)$ & $6.1 \times 10^{-3}[127,128]$ \\
$B r(h \rightarrow e \mu)$ & $6.2 \times 10^{-5}[132]$ \\
\hline
\end{tabular}

Table 2. Current experimental situation of LFV Higgs decays.

of the Higgs boson and on the associated Yukawa couplings are the most stringent to date (see table 2). Similarly, the null search results of $\operatorname{Br}(\tau \rightarrow e / \mu+\gamma)$ and $\operatorname{Br}(\tau \rightarrow 3 e / \mu)$ [129] translate into bounds on corresponding objects like $\sqrt{Y_{i j}^{2}+Y_{j i}^{2}}[130]$.

Limits on LFV Higgs decay processes closely follow the search results of $h \rightarrow \mu \mu / \tau \tau$ channels. Evidence for the $125 \mathrm{GeV}$ Higgs boson decaying to a pair of $\tau$ (or $\mu$ ) leptons are presented in refs. [133-136]. Furthermore, dedicated searches are conducted for additional neutral Higgs bosons decaying to the $\tau^{+} \tau^{-}$final state in proton-proton collisions at the $13 \mathrm{TeV}$ LHC [137-139] which lead exclusion plots in the $m_{A}-\tan \beta$ plane and also give limits on $\sigma(g g \rightarrow \phi) \times \operatorname{Br}\left(\phi \rightarrow \tau^{+} \tau^{-}\right)$with $(\phi=h, H, A)$. Since the cLFV processes of heavier Higgs bosons are proportional to $B r\left(\phi \rightarrow \tau^{+} \tau^{-}\right)$[49] these limits are extremely important for any analysis of $\operatorname{Br}(\phi \rightarrow \mu \tau)$. Similar results for $\tau^{+} \tau^{-}$finals states at $\sqrt{s}=8 \mathrm{TeV}$ are available in [140, 141]. Recently, some model independent analyses of heavier Higgs boson decaying into $\mu \tau$ channel have been performed [142] and it is shown that, at $\sqrt{s}=14 \mathrm{TeV}$ with $\mathcal{L}=300 \mathrm{fb}^{-1}$ the sensitivities to the experimental probes increase with heavier Higgs boson masses. Lepton flavor violating decays of Higgs have also been searched for in the first-second and first-third generations of leptons, i.e $e \mu$ and $e \tau$ [131, 143] channels in the LHC at $\sqrt{s}=8 \mathrm{TeV}$. These classes of LFV processes are also being studied in LHC through the decays of neutral heavy Higgs like bosons for different supersymmetric and non-supersymmetric models [144-146].

\section{Results}

We divide our LFV decay analyses into three parts namely, $l_{i} \rightarrow l_{j} \gamma, l_{i} \rightarrow 3 l_{j}$ and $\phi(=h, H, A) \rightarrow l_{i} \bar{l}_{j}$. As mentioned before, we try to explore the LFV effects of the relevant trilinear coupling matrices related to the holomorphic and non-holomorphic trilinear interactions. Simply, out of their association with the type of interactions we will label the couplings themselves as "holomorphic" (like $A_{i j}$ or $A_{i i} \equiv A_{i}$ ) or "non-holomorphic" (like $A_{i j}^{\prime}$ or $A_{i i}^{\prime} \equiv A_{i}^{\prime}$ ). Furthermore, as pointed out earlier, for simplicity, our analysis probes either (i) a non-vanishing set of $\left[A_{i j}, A_{i}\right]$ while having vanishing values for the set $\left[A_{i j}^{\prime}, A_{i}^{\prime}\right]$ or (ii) vice-versa. We will particularly identify the region of parameter space in relation to a given constraint from an LFV decay where non-holomorphic couplings may have prominent roles. Considering the fact that only the trilinear couplings associated with sleptons are of importance in this analysis, we will either consider the matrices $A_{f}=0$ and $A_{f}^{\prime} \neq 0$ and vice-versa, where $f \equiv e, \mu, \tau$. Combining the holomorphic and non-holomorphic couplings, we will often use $A_{f}^{(\prime)}$ to mean either $A_{f}$ or $A_{f}^{\prime}$ depending on the context. We vary both 


\begin{tabular}{|c|c|c|c|}
\hline Parameters & Value & Parameters & Value \\
\hline$M_{1}$ & {$[100,1000]$} & $M_{2}$ & 1500 \\
$M_{3}$ & 2800 & $\mu$ & 800 \\
$m_{A}$ & 1500 & $\tan \beta$ & 40 \\
$M_{\tilde{q}_{33}} / M_{\tilde{u}_{33}}$ & 2000 & $M_{\tilde{d}_{33}}$ & 2000 \\
$M_{\tilde{q}_{11,22}} / M_{\tilde{u}_{11,22}}$ & 2000 & $M_{\tilde{d}_{11,22}}$ & 2000 \\
$M_{\tilde{L}_{11,22,33}}$ & {$[1000,10000]$} & $M_{\tilde{e}_{11,22,33}}$ & {$[1000,10000]$} \\
\hline$A_{t}, A_{b}$ & $-2200,0$ & $A_{t}^{\prime}, A_{b}^{\prime}$ & 0,0 \\
\hline$A_{(e, \mu, \tau)},\left|\delta_{i j}\right|<1$ & {$[-8000,8000]$} & $A_{(e, \mu, \tau)}^{\prime},\left|\delta_{i j}^{\prime}\right|<1$ & {$[-8000,8000]$} \\
\hline
\end{tabular}

Table 3. Soft masses and trilinear parameters are listed here. All the masses and trilinear couplings are in $\mathrm{GeV}$ and there is no off-diagonal entries in the soft bilinear mass matrices.

the diagonal and off-diagonal components of $\mathbf{A}^{(\prime)}$ eij over broad range of values (see table 3) subject to the condition $\left|\delta_{i j}^{(\prime)}\right|<1$ with $i \neq j$, where $\delta_{i j}^{(\prime)}=\frac{A^{(\prime)} e_{i j}}{A^{(\prime)} e_{i i}}$. Here, we remind that all the off-diagonal entries of the slepton mass matrices are considered to be zero leading to flavor violation possible only from the trilinear coupling sources. We will impose the charge breaking constraint and select the possible diagonal and off-diagonal trilinear coupling values that would obey eq. (3.11). We must emphasize here that in an analysis that scans the diagonal and off-diagonal elements of the trilinear coupling matrices, such as the present one, we do not expect severe restrictions on the off-diagonal entries $A_{i j}^{(\prime)}$ simply because of the possible cancellation of terms in the left hand side of eq. (3.11). On the other hand, a choice of fixed signs of diagonal and off-diagonal entries of tri-linear coupling matrix elements would show prominent effects of charge breaking. Apart from the above, we impose the experimental bounds of different cLFV observables and LHC direct search results for $\phi \rightarrow l_{i} \bar{l}_{j}$. The values/ranges of relevant soft parameters used in this analysis are listed in table 3. We compute the SUSY mass spectra and related branching fractions from SARAH (v4.10.0) [147, 148] generated FORTRAN codes that are subsequently used in SPheno (v4.0.3) [149]. All the flavor observables are calculated using FlavorKit which is inbuilt within the SARAH-SPheno framework. In regard to a few relevant SM parameters, we use $m_{t}^{\text {pole }}=173.5 \mathrm{GeV}, m_{b}^{\overline{M S}}=4.18 \mathrm{GeV}$ and $m_{\tau}=1.77 \mathrm{GeV}$ [150] and we use the SUSY mass scale as $M_{\mathrm{SUSY}}=\sqrt{m_{\tilde{t}_{1}} m_{\tilde{t}_{2}}}$. We further impose the following limits for Higgs mass $m_{h},{ }^{7}$ branching ratios like $\operatorname{Br}(b \rightarrow s \gamma)$ and $\operatorname{Br}\left(B_{s} \rightarrow \mu^{+} \mu^{-}\right)$at $2 \sigma$ level, lighter chargino mass bound from LEP, along with an LHC limit for the direct lighter top squark

\footnotetext{
${ }^{7}$ We consider a $\pm 3 \mathrm{GeV}$ theoretical uncertainty in computing lighter Higgs mass [151-157] that arises from the uncertainties from radiative corrections up to three loops, top quark pole mass, renormalization scheme and scale dependencies etc.
} 
searches $[150,158]$.

$$
\begin{aligned}
122.1 \mathrm{GeV} & \leqslant m_{h} \leqslant 128.1 \mathrm{GeV} \\
2.99 \times 10^{-4} & \leqslant B r(b \rightarrow s \gamma) \leqslant 3.87 \times 10^{-4} \\
1.5 \times 10^{-9} & \leqslant B r\left(B_{s} \rightarrow \mu^{+} \mu^{-}\right) \leqslant 4.3 \times 10^{-9} \\
m_{\tilde{\chi}_{1}}^{ \pm} & \geq 104 \mathrm{GeV}, \quad m_{\tilde{t}_{1}} \geq 1000 \mathrm{GeV} .
\end{aligned}
$$

\section{$5.1 \quad l_{j} \rightarrow l_{i} \gamma$}

We show the results of computation of $\operatorname{Br}\left(l_{j} \rightarrow l_{i} \gamma\right)$ in figure 4 . Here, the cyan and blue colored zones correspond to the scanning selected for the relevant holomorphic and non-holomorphic trilinear coupling matrix elements $A_{i j}$ and $A_{i j}^{\prime}$ respectively. Only the parameter points that satisfy the charge breaking vacuum constraint of eq. (3.11) are shown where the domain of variations of $A_{i j}$ and $A_{i j}^{\prime}$ are mentioned in table 3 . Apart from $A_{i j}^{(\prime)}$, the mass parameters varied are $M_{1}$, the mass of bino and the diagonal soft masses of the left and right handed sleptons namely, $M_{\tilde{L}_{i i}}$ and $M_{\tilde{e}_{i i}}$ whose ranges are as given in table 3 . As we mentioned earlier, in this analysis we do not consider any non-vanishing off-diagonal entry for the scalar mass matrices. The off-diagonal trilinear couplings $A_{e \mu}^{\prime}$ has stronger influence on $\operatorname{Br}(\mu \rightarrow e \gamma)$ compared to $A_{e \mu}$. The resulting influence superseed the present upper bound of $\operatorname{Br}(\mu \rightarrow e \gamma)$ (see figure $4 \mathrm{a})$. The excluded regions that are unavailable via experimental limits are shown as gray bands in the top. Additionally, the black horizontal lines in figure $4 \mathrm{a}$ to figure $4 \mathrm{c}$ display the upcoming sensitivities for the respective channels of $l_{j} \rightarrow l_{i} \gamma$ (see table 1 ). We remind that the effect of a non-vanishing $A_{e \mu}^{\prime}$ is associated with an enhancement by $\tan \beta$ that in general pushes up the branching ratio $B r(\mu \rightarrow e \gamma)$ over 2 to 3 orders of magnitude compared to the same arising out of $A_{e \mu}$. This is also true for the other decay modes namely $\tau \rightarrow e \gamma$ and $\tau \rightarrow \mu \gamma$ in relation to the corresponding trilinear couplings. However, only the present $\operatorname{Br}(\mu \rightarrow e \gamma)$ bound is strong enough to exclude a significant amount of NHSSM parameter space depending on the values of $A_{e \mu}^{\prime}$ and the mass parameters that would simultaneously satisfy the charge breaking constraint.

\section{$5.2 l_{j} \rightarrow 3 l_{i}$}

We will now try to investigate the influence of non-vanishing trilinear couplings $A_{i j}$ and $A_{i j}^{\prime}$ on $\operatorname{Br}\left(l_{j} \rightarrow 3 l_{i}\right)$ in figure 5 . This is in spite of the fact that any influence is yet too far to be tested in experiments such as that for $\operatorname{Br}(\tau \rightarrow 3 e)$ and $\operatorname{Br}(\tau \rightarrow 3 \mu)$. Similar to figure 4 , the cyan and blue colored regions refer to parameter points with given $A_{i j}$ and $A_{i j}^{\prime}$ values respectively that would avoid the charge breaking minima. The present bounds of $\operatorname{Br}(\tau \rightarrow 3 e)$ and $\operatorname{Br}(\tau \rightarrow 3 \mu)$ are of the order of $10^{-8}$ by BELLE collaboration [88]. These are expected to reach $10^{-9}$ in Super B [121]. Clearly as seen in the figures both the limits are significantly larger compared to the level of contributions under discussion. Regarding $\operatorname{Br}(\mu \rightarrow 3 e)$, a non-vanishing $A_{e \mu}^{\prime}$ can push it up to $10^{-12}$ but $\operatorname{Br}(\mu \rightarrow e \gamma)$ is more effective a constraint to limit $A_{e \mu}^{\prime}$.

In figure 5 we see the stretch of $\operatorname{Br}(\tau \rightarrow 3 e / \mu)$ with holomorphic and non-holomorphic trilinear couplings respectively. The color coding is the same as in the figure 4 . The cyan 


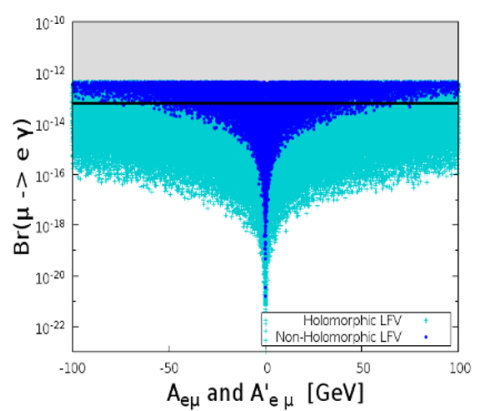

(a)

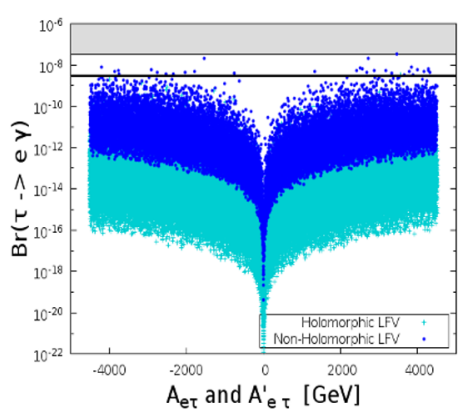

(b)

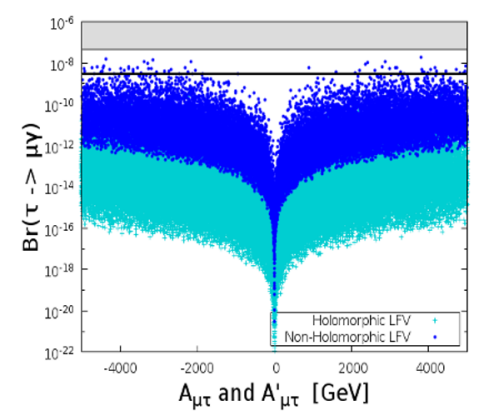

(c)

Figure 4. Branching ratios of $l_{j} \rightarrow l_{i} \gamma$ as a function of $A_{i j}$ in cyan and $A_{i j}^{\prime}$ in blue after satisfying the charge breaking bound and are checked through the other collider bounds viz. eq. (5.1) and table 1. The gray shaded regions and black horizontal lines in each plot denote the present exclusion region and future announced sensitivity of the corresponding channels.

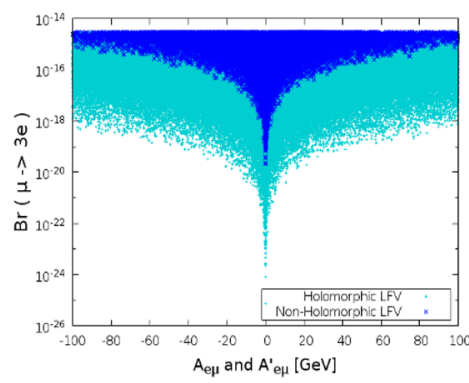

(a)

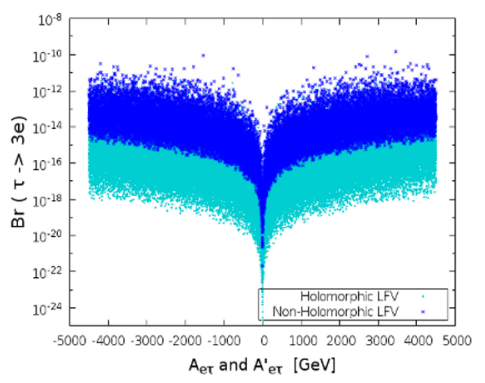

(b)

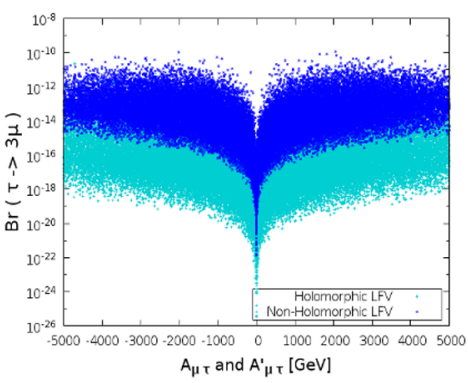

(c)

Figure 5. The variation of $\operatorname{Br}(\mu \rightarrow 3 e)$ with $A_{e \mu}$ and $A_{e \mu}^{\prime}$ has been shown in 5(a), 5(b) depicts $\operatorname{Br}(\tau \rightarrow 3 e)$ with $A_{e \tau}$ and $A_{e \tau}^{\prime}$ and in $5(\mathrm{c})$ depicts $\operatorname{Br}(\tau \rightarrow 3 \mu)$ with $A_{\mu \tau}$ and $A_{\mu \tau}^{\prime}$. They are passed through the checks of (5.1) and table 1 , and after avoiding the overall charge breaking minima given in (3.8) and (3.11) for holomorphic and non-holomorphic trilinear couplings respectively.

and blue colored points are shown after avoiding the charge breaking minima. The current sensitivity of these two channels is of the order of $10^{-8}$ by BELLE collaboration [88] which is expected to reach $10^{-9}$ in Super B [121]. In the following, after satisfying all the respective bounds as mentioned in eq. (5.1) and in table 1 and the radiative decays $\operatorname{Br}(\tau \rightarrow e \gamma), \operatorname{Br}(\tau \rightarrow \mu \gamma)$ in particular, we find both $\operatorname{Br}(\tau \rightarrow 3 e)$ and $\operatorname{Br}(\tau \rightarrow 3 \mu)$ can reach up to $\sim 10^{-11}$. This is again two orders of magnitude smaller than the future proposed sensitivity. The maximum reaches are $10^{-10}$ and $10^{-11}$ for $\operatorname{Br}(\tau \rightarrow 3 e)$ and $\operatorname{Br}(\tau \rightarrow 3 \mu)$ respectively, while for $\operatorname{Br}(\mu \rightarrow 3 e)$ may become $10^{-12}$ with non-holomorphic $A_{e \mu}^{\prime} . \operatorname{Br}(\mu \rightarrow e \gamma)$ limits the free increase of $\operatorname{Br}(\mu \rightarrow 3 e)$ in particular.

We like to comment that virtual Higgs exchange can also induce $\tau$ decaying to $\mu$ along with psedoscalar meson like $\tau \rightarrow \mu\left(\pi / \eta / \eta^{\prime}\right)$ though the later decay fractions lie much below the future sensitivity presented in table 1 . With CP conservation in Higgs sector, only the exchange of CP-odd Higgs is expected to be present dominantly because of its enhanced 
couplings to the down-type quarks. These interactions and relations to the other LFV processes can be found in $[50,159]$.

\section{$5.3 \phi(h, H, A) \rightarrow l_{i} \bar{l}_{j}$}

We focus now on flavor violating Higgs decays that lead to two oppositely charged leptons where a Higgs boson can be the SM-like Higgs boson $(h)$, CP-even heavier Higgs $(H)$ or CPodd Higgs $(A)$. We however note that the current experimental level to probe light higgs decay branching ratios as seen in table 2 is way too large compared to the branching ratio values shown below. This is unlike the decays of the heavier Higgs where non-holomorphic parameters may give large contributions.

- $\mathbf{h} \rightarrow \mathbf{l}_{\mathbf{i}} \overline{\mathbf{l}}_{\mathbf{j}}$. We discuss the light higgs decay here for completeness before moving to the discussion of heavy Higgs leptonic decays with flavor violations. Figure 6 shows the scatter plots of the branching ratios $B r(h \rightarrow e \mu), B r(h \rightarrow e \tau)$ and $B r(h \rightarrow \mu \tau)$ when $A_{e \mu}^{\left({ }^{\prime}\right)}, A_{e \tau}^{\left({ }^{\prime}\right)}$ and $A_{\mu \tau}^{\left({ }^{\prime}\right)}$ respectively are varied. All the points satisfy the mass bounds and flavor constraints of eq. (5.1) and table 1 apart from avoiding any charge breaking minima (eqs. (3.8), (3.11)). The color convention is same as before, i.e. the points in blue and cyan are for varying nonholomorphic and holomorphic trilinear couplings respectively. The spread in the colored points is the consequence of the random scanning of soft masses as mentioned in table 3 . One notes that, irrespective of the source of flavor violation the branching ratio $\operatorname{Br}(\phi \rightarrow$ $l_{i} \bar{l}_{j}$ ) itself is proportional to $\tan ^{2} \beta[49]$. An appropriate $\mathrm{NH}$ coupling additionally multiplies the result with $\tan ^{2} \beta$ potentially leading to an enhancement by a factor of $\sim 10^{3}$ when compared to corresponding the MSSM scenario for $\tan \beta \gtrsim 30$. Our results for the holomorphic case closely agree with the results of [67] with LR type flavor violation in the slepton sector of MSSM. $\operatorname{Br}(h \rightarrow e \tau)$ is in the ballpark of $\sim 10^{-10}\left(\sim 10^{-13}\right)$ for non(-holomorphic) trilinear parameters. We note that as seen in figure 4(a), stringency due to $\operatorname{Br}(\mu \rightarrow e \gamma)$ causes the extent of allowed variation $A_{e \mu}^{(\prime)}$ to be much smaller than the other trilinear couplings corresponding to those with $e-\tau$ or $\mu-\tau$. Consequently, $\operatorname{Br}(h \rightarrow e \mu)$ of figure 10a is smaller by 3 to 4 orders of magnitude with respect to the branching ratios of figure 10b and figure 10c.

We will now briefly study the level of dependence of the associated sparticle masses on the above LFV $h$-decays in figure 7 . The relevant loops contain neutralinos and sleptons. The left and the right panels show $\operatorname{Br}(h \rightarrow \mu \tau)$ when soft masses of bino $\left(M_{1}\right)$ and the lighter stau $\left(m_{\tilde{\tau}_{1}}\right)$ respectively are varied. All the input parameters and ranges are as given in table 3. The branching ratio profile of figure $7 \mathrm{~b}$ shows that the radiative corrections associated with the decay expectedly fall with $m_{\tilde{\tau}_{1}}$ while the same of figure 7 a hardly shows any correlation with $M_{1}$. We note that for NHSSM, the regions of larger $\operatorname{Br}(h \rightarrow \mu \tau)$ that are typically associated with large $A_{\mu \tau}^{\prime}$ may lead to tachyonic staus due to large L-R slepton mixing. This is consistent with what we find to be a small discarded zone with $m_{\tilde{\tau}_{1}} \sim$ near $1 \mathrm{TeV}$ that could otherwise have a large branching ratio.

- $\mathbf{H} / \mathbf{A} \rightarrow \mathbf{l}_{\mathbf{i}} \overline{\mathbf{l}}_{\mathbf{j}}$. We now turn to explore the dependence on $A_{i j}^{\prime}$ 's when heavier Higgs bosons decay into leptons with LFV namely, $H / A \rightarrow l_{i} \bar{l}_{j}$. Figure $8(\mathrm{a})$, figure $8(\mathrm{~b})$ and figure $8(\mathrm{c})$ refer to the scatter plots of the associated branching ratios for the decay of

$\mathrm{H}$ or A bosons into $e \mu, e \tau$ and $\mu \tau$ respectively where $A_{i j}^{\prime}$ are varied. The figures have 


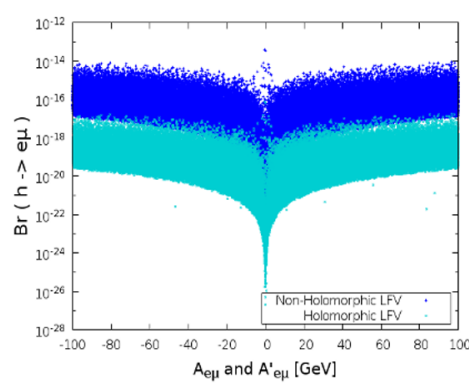

(a)

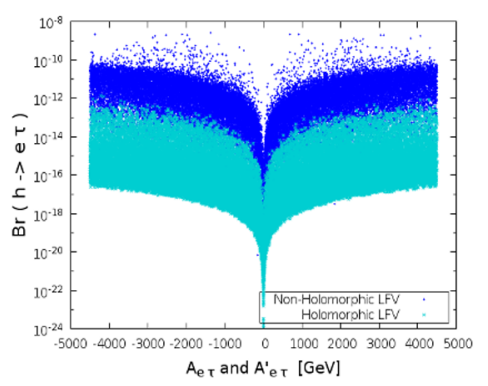

(b)

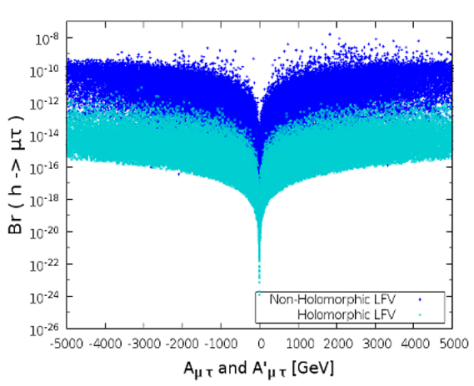

(c)

Figure 6. Figure 6(a) shows the variation of $B r(h \rightarrow e \mu)$ with $A_{e \mu}$ and $A_{e \mu}^{\prime}$, in figure 6(b) dependence of $\operatorname{Br}(h \rightarrow e \tau)$ with $A_{e \tau}$ and $A_{e \tau}^{\prime}$ and 6(c) shows the variation of $\operatorname{Br}(h \rightarrow \mu \tau)$ with $A_{\mu \tau}$ and $A_{\mu \tau}^{\prime}$. All points fulfill the analytical expressions of charge breaking minima given in (3.8) and (3.11) and the color coding is same as previous.

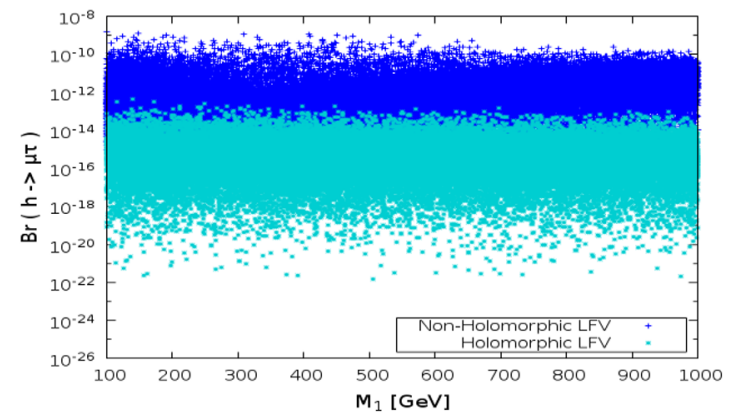

(a)

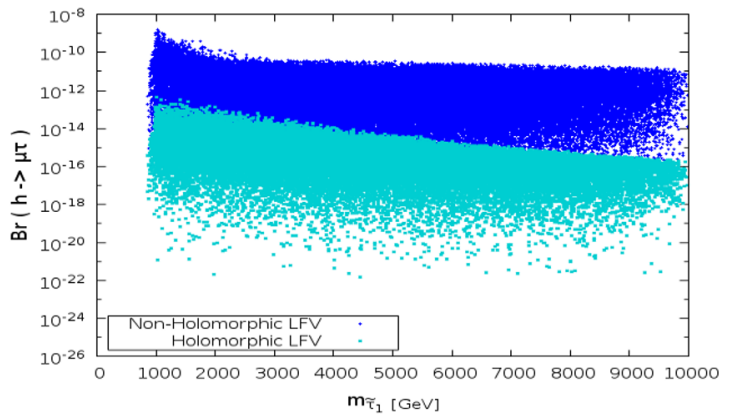

(b)

Figure 7. In left panel of figure 7 we can see the dependence of $\operatorname{Br}(h \rightarrow \mu \tau)$ on $M_{1}$. In the right side the same branching ratio is plotted against the lightest slepton mass. All other soft mass parameters and trilinear couplings are according to table 3 and are checked through the charge breaking minima condition.

the same color convention as before. Here, all the points satisfy the mass bounds and flavor constraints of eq. (5.1) and table 1. As before, the figures show only the points that satisfy the charge breaking minima constraints (eqs. (3.7), (3.11)) for varying holomorphic or non-holomorphic trilinear coupling parameters. The branching ratios are expected to be large because the couplings of $\mathrm{H} / \mathrm{A}$ to down-type fermions grow with $\tan \beta$. As we discussed earlier, the LFV branching fraction $\operatorname{Br}\left(\phi_{k} \rightarrow e \tau\right)$ or $\operatorname{Br}\left(\phi_{k} \rightarrow \mu \tau\right)$ can be cast in terms of flavor conserving di-tau branching ratio $B r\left(\phi_{k} \rightarrow \tau \tau\right)$ following eq. (2.20) where $\phi_{k}$ refers to $h, H, A$ for $k=1,2,3$ respectively. $B r\left(\phi_{k} \rightarrow \tau \tau\right)$ in general depends on slepton and neutralino masses [49] with hardly any dependence on trilinear couplings at the lowest order. We must use the LHC data here, particularly for the heavier Higgs bosons decaying into the di-tau channels $[138,160]$. The constraint in the $\left[m_{A}, \tan \beta\right]$ plane is rather stringent in the large $\tan \beta$ and small $m_{A}$ region. With our choice of $m_{A}$, that is rather high, the flavor conserving branching ratio satisfies the LHC limit [138]. Apart from 


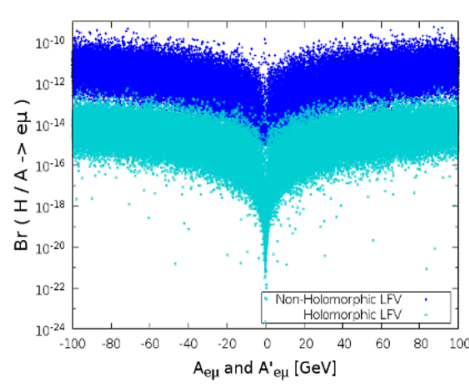

(a)

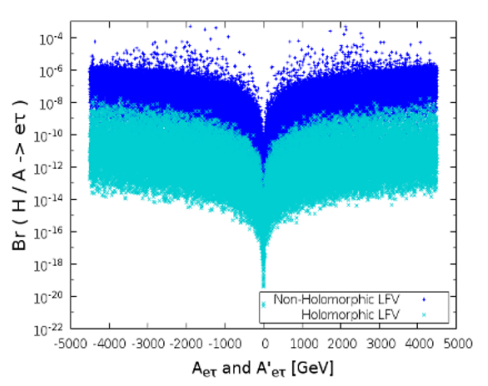

(b)

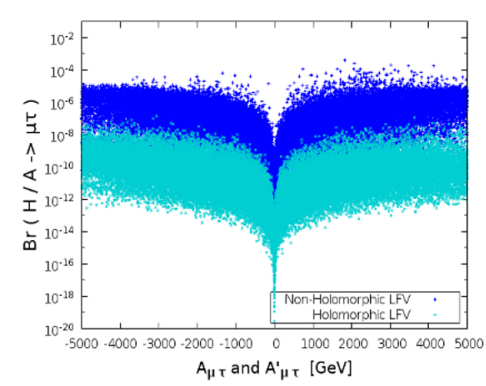

(c)

Figure 8. $\operatorname{Br}(H / A \rightarrow e \mu)$ as a function of $A_{e \mu}^{\left({ }^{\prime}\right)}$ in the left most side, $B r(H / A \rightarrow e \tau)$ as a function of $A_{e \tau}^{\left({ }^{\prime}\right)}$ in the middle and $B r(H / A \rightarrow \mu \tau)$ as a function of $A_{\mu \tau}^{\left({ }^{\prime}\right)}$ in the right side are shown with $m_{H}, m_{A}=1.5 \mathrm{TeV}$. Color coding is same as explained in figure 6 .

this, our computed results of $\sigma\left(g g \phi_{k}\right) \operatorname{Br}\left(\phi_{k} \rightarrow \tau \tau\right)$ for all $k$ fall in the allowed zones of the LHC limit [160]. The flavor violating decay rates of $H / A$ that are computed by using eq. (2.20) become large because of large $C_{H}$ or $C_{A}$ (eq. (2.21)) when $h$ is chosen to be SM like in its couplings. The decay rates may potentially get amplified by a factor of $\sim 10^{3}$ (via $\propto \tan ^{2} \beta$ ) in presence of non-vanishing non-holomorphic trilinear couplings. Thus, unlike the case of LFV $h$-decay of figure 6 , the LFV branching ratios of $H / A$ as shown in figures $8(\mathrm{~b})$ and $8(\mathrm{c})$ may scale as high as $10^{-4}$. This may be of significance in relation to a future high energy collider.

\subsection{Direct constraints on $Y_{i j}$ from LFV Higgs decay limits of LHC}

With identical Yukawa couplings that MSSM inherits from SM, we will now use the constraints on relevant off-diagonal Yukawa couplings arising out of the null limits of SM-like higgs decays like $h \rightarrow \mu \tau, h \rightarrow e \tau$, and $h \rightarrow e \mu$ as given by the LHC data. The CMS collaboration performed direct exploration of $h \rightarrow \mu \tau$, followed by the hunt for $h \rightarrow e \tau / e \mu$ decays with $8 \mathrm{TeV}$ corresponding to an integrated luminosity of $19.7 \mathrm{fb}^{-1}[124,131]$. The hadronic, electronic and muonic decay channels for the $\tau$-leptons were also explored for the above mentioned LFV processes with $13 \mathrm{TeV}$ LHC data $[127,128,132]$. The null results can effectively put upper limits on the off-diagonal namely $\mu \tau$ and $e \tau$ Yukawa couplings. We relate this to the outcome of having non-vanishing Yukawa couplings arising out of radiative corrections due to the trilinear soft terms of both holomorphic and nonholomorphic origins. The current LHC bounds $[127,128]$ are $\sqrt{\left(Y_{\mu \tau}^{2}+Y_{\tau \mu}^{2}\right)}<1.50 \times 10^{-3}$ and $\sqrt{\left(Y_{e \tau}^{2}+Y_{\tau e}^{2}\right)}<2.26 \times 10^{-3}$. Regarding $Y_{e \mu}$, one finds that the null observation of $\mu \rightarrow e \gamma$ implies a very stringent limit: $\sqrt{\left(Y_{e \mu}^{2}+Y_{\mu e}^{2}\right)}<3.6 \times 10^{-6}$ [130]. We note that in general for an LFV scenario there can be two independent Yukawa couplings $Y_{i j}$ and $Y_{j i}$ [161]. For example, this arises from different possible Yukawa couplings with higgs for $e_{L}$ with $\mu_{R}$ and $\mu_{L}$ with $e_{R}$ superfields. However, we consider them to be identical in this analysis for simplicity and this is consistent with our assumption of a single trilinear soft parameter $A_{i j}$ which is same as $A_{j i}$. 


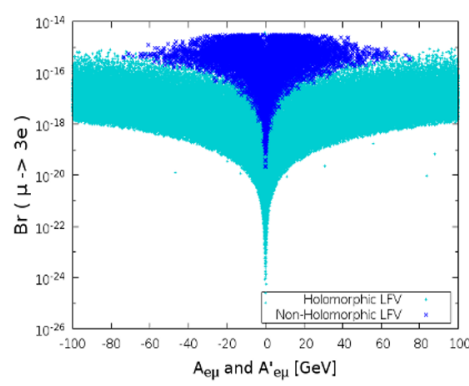

(a)

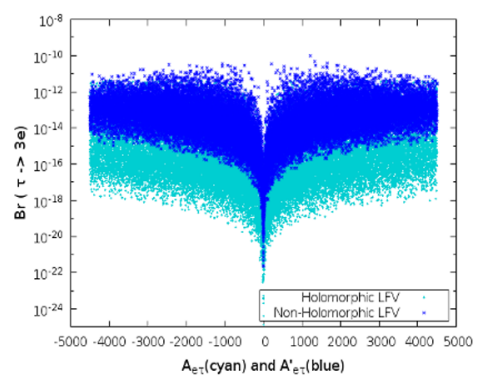

(b)

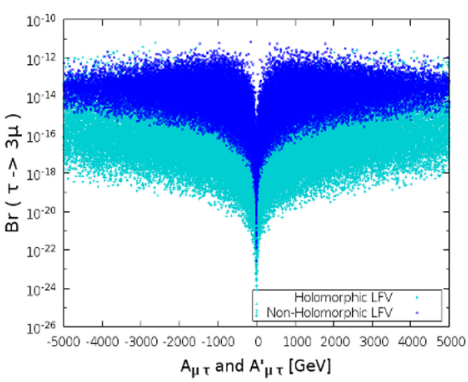

(c)

Figure 9. Effect of imposing the LHC constraints on $Y_{i j}$ on the analysis of figure 5: scatter plots of $\operatorname{Br}(\mu \rightarrow 3 e)$ with $A_{e \mu}$ and $A_{e \mu}^{\prime}$ are shown in $9(\mathrm{a})$. Similar plots for $\operatorname{Br}(\tau \rightarrow 3 e)$ with $A_{e \tau}$ and $A_{e \tau}^{\prime}$ are shown in figure $9(\mathrm{~b})$. Figure $9(\mathrm{c})$ is for $\operatorname{Br}(\tau \rightarrow 3 \mu)$ with $A_{\mu \tau}$ and $A_{\mu \tau}^{\prime}$.

\subsubsection{Results for varying bino and slepton masses}

We now impose the Yukawa coupling bounds to update figures 5, 6 and 8 keeping all other constraints unchanged. This will help us in understanding the extent of influence of the above constraints on the trilinear soft paramaters. The color conventions in these figures are same as before. Below, we will only emphasize the essential differences between the figures focusing only on NHSSM. This is simply because the above bounds are hardly effective for MSSM involving only the trilinear holomorphic couplings.

Compared to figure 5a, figure 9a discards a significant amount of large $\operatorname{Br}(\mu \rightarrow 3 e)$ zone with large $\left|A_{e \mu}^{\prime}\right|$. Thus, approximately a region with $\left|A_{e \mu}^{\prime}\right|$ above $60 \mathrm{GeV}$ is eliminated. The above only demonstrate the stringency of the constraints on the first two generations of the off-diagonal Yukawa couplings arising from the null observation of $\mu \rightarrow$ er [130] along with the CMS results on the first two generations [132, 162]. Figure 9b is not so different from figure 5b indicating only an insignifant influence. Regarding figure $9 \mathrm{c}$ vs figure 5c we see that the upper limit of $\operatorname{Br}(\tau \rightarrow 3 \mu)$ is truncated by approximately an order of magnitude over the parameter space, still being a mild effect.

The effect of the off-diagonal Yukawa coupling constraints from the CMS results is shown for the cLFV $h$-decays in figure 10 in which the scattered plots of the two colors for each of the sub-figures are subsets of the same of figure 6 . In the context of limiting $A_{i j}^{\prime}$ the conclusion is essentially similar to the discussion made in relation to each of the sub-figures of figure 9 .

Coming to cLFV $H$-decays, each of the scattered plots of $\operatorname{Br}\left(H / A \rightarrow l_{i} \bar{l}_{j}\right)$ vs $A_{i j}$ and $A_{i j}^{\prime}$ as shown in figure 11 incorporates the off-diagonal Yukawa coupling constraints and these are subsets of the appropriate sub-figures of figure 8. Unlike before $\left|A_{e \mu}^{\prime}\right|$ is further limited, down to $30 \mathrm{GeV}$ as may be seen in figure 11a, signifying an appreciable degree of constraint when compared with figure $9 \mathrm{a}$ or $10 \mathrm{a}$. While figure $11 \mathrm{~b}$ is not much different from figure $8 \mathrm{~b}$, the upper limit of $\operatorname{Br}(H / A \rightarrow \mu \tau)$ in figure 11c is approximately reduced by an order of magnitude over the parameter space when compared with figure $8 \mathrm{c}$. 


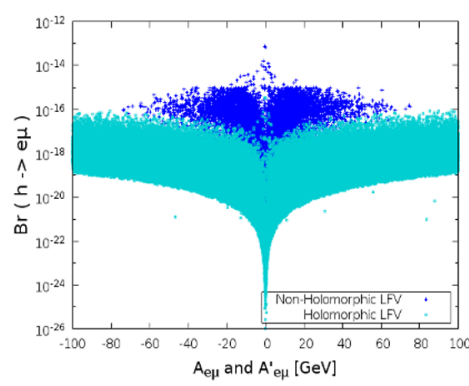

(a)

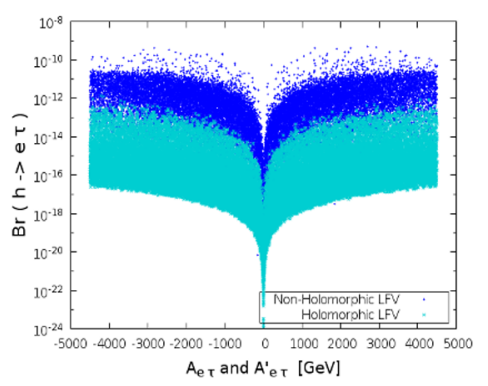

(b)

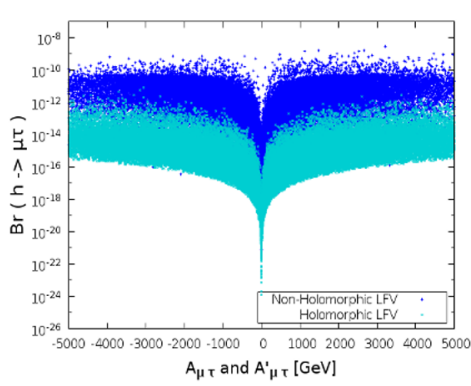

(c)

Figure 10. Effect of imposing the LHC constraints on $Y_{i j}$ on the analysis of figure 6: scatter plots of $\operatorname{Br}(h \rightarrow e \mu)$ with $A_{e \mu}$ and $A_{e \mu}^{\prime}$ are shown in 10(a). Similar plots for $\operatorname{Br}(h \rightarrow e \tau)$ with $A_{e \tau}$ and $A_{e \tau}^{\prime}$ are shown in figure 10(b). Figure 10(c) is for $\operatorname{Br}(h \rightarrow \mu \tau)$ with $A_{\mu \tau}$ and $A_{\mu \tau}^{\prime}$.

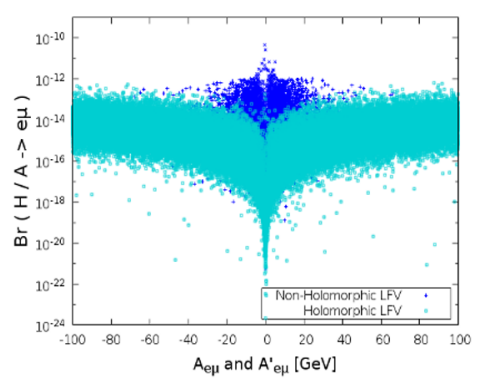

(a)

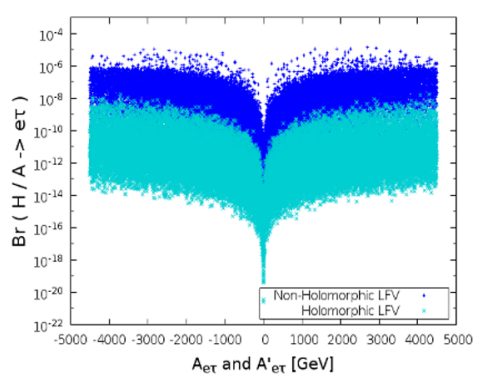

(b)

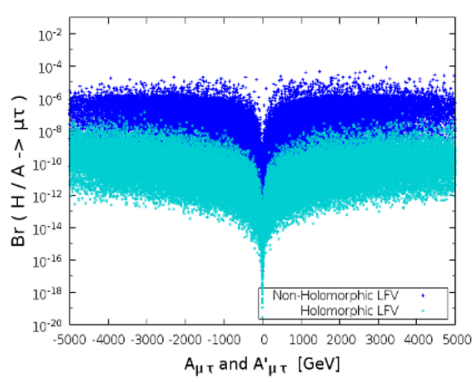

(c)

Figure 11. Effect of imposing the LHC constraints on $Y_{i j}$ on the analysis of figure 8: scatter plots of $\operatorname{Br}(H / A \rightarrow e \mu)$ with $A_{e \mu}$ and $A_{e \mu}^{\prime}$ are shown in 11(a). Similar plots for $\operatorname{Br}(H / A \rightarrow e \tau)$ with $A_{e \tau}$ and $A_{e \tau}^{\prime}$ are shown in figure 11(b). Figure 11(c) is for $B r(H / A \rightarrow \mu \tau)$ and $A_{\mu \tau}^{\prime}$.

\subsubsection{Results for fixed bino and slepton masses}

We will see now that the multi-parameter scattered plots like figure 9 to 11 are quite limited in emphasizing the degree of importance of the Yukawa coupling bounds. Hence, in this subsection we fix $M_{1}$ at $400 \mathrm{GeV}$ and consider the diagonal soft slepton soft mass parameters $\left(M_{\tilde{L}} \& M_{\tilde{e}}\right)$ to have the specific values 1, 2, 3 and $5 \mathrm{TeV}$ for MSSM and 2, 3 and $5 \mathrm{TeV}$ for NHSSM. The resulting figure 12 depicts the behavior of $\sqrt{Y_{i j}^{2}+Y_{j i}^{2}}$ with $A_{i j}$ or $A_{i j}^{\left({ }^{\prime}\right)}$ corresponding to the MSSM and NHSSM cases respectively. In the left panel we show the plots for the holomorphic off-diagonal trilinear terms and in the right panel we show the similar terms for the non-holomorphic ones. Clearly, larger radiative corrections are induced in the case of non-vanishing $A_{i j}^{\prime}$, particularly, when sleptons are light. For smaller soft masses of sleptons $M_{\tilde{L}} \& M_{\tilde{e}} \simeq 1 \mathrm{TeV}$, with our choice of high $\tan \beta$, non holomorphic trilinear couplings may even generate unacceptable tachyonic states of sleptons. The black horizontal lines in each plot relates to the upper bound on respective $\sqrt{Y_{i j}^{2}+Y_{j i}^{2}}$. As we will see next that for the first two generations, the black line corresponds to the upper 

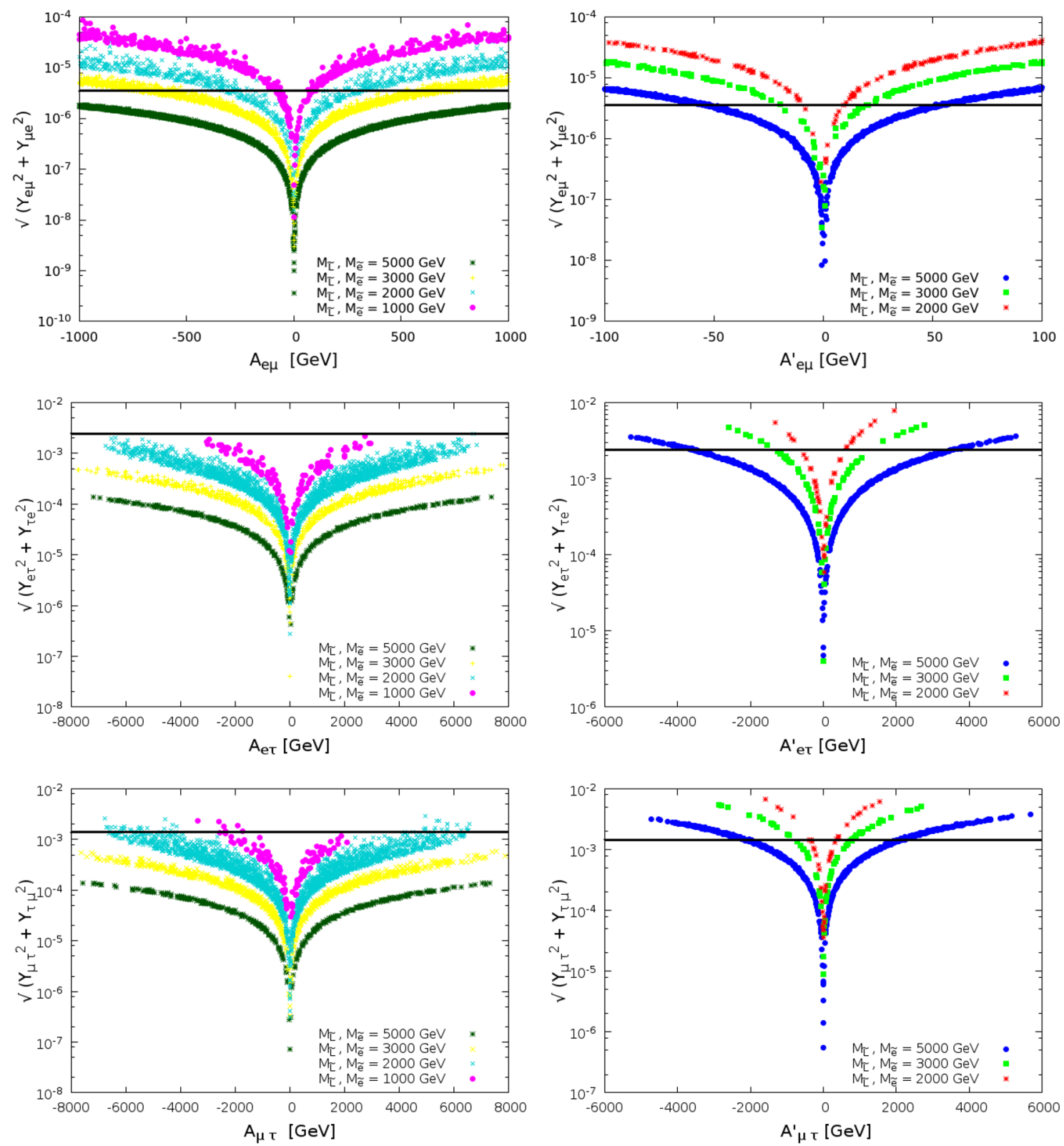

Figure 12. $\sqrt{Y_{i j}^{2}+Y_{j i}^{2}}$ vs. $A_{i j}$ (left panel) and $A_{i j}^{\prime}$ (right panel) are shown for fixed slepton bilinear soft masses and $M_{1}=400 \mathrm{GeV}$. Other fixed parameters are as stated in table 3. For holomorphic trilinear couplings, slepton soft masses are fixed at 1,2,3 and $5 \mathrm{TeV}$ and the same for $\mathrm{NH}$ couplings are at 2,3 and $5 \mathrm{TeV}$. Black horizontal lines in each plot denote the corresponding upper limits on the off-diagonal Yukawa couplings.

limit of $\operatorname{Br}(\mu \rightarrow e \gamma)$, and for the other two generations they refer to the null results of $\operatorname{Br}\left(h \rightarrow l_{i} \bar{l}_{j}\right)$ searched by the LHC experiment.

With the understanding on how off-diagonal Yukawa couplings can be directly influenced by trilinear parameters, we now present figure 13 that shows the derived bounds on 


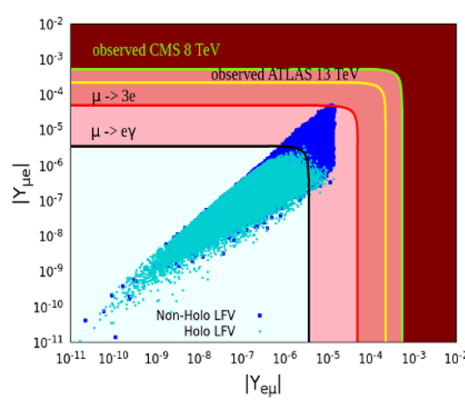

(a)

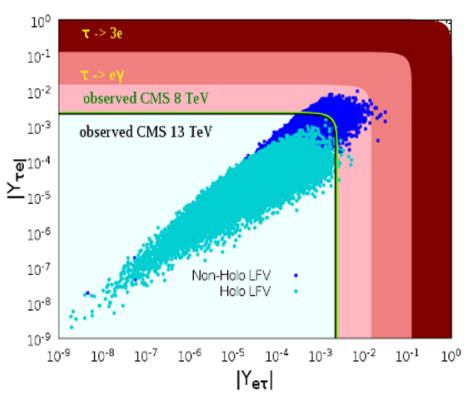

(b)

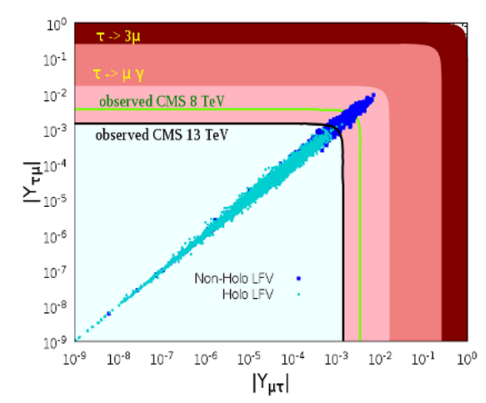

(c)

Figure 13. Constraints on the flavour violating Yukawa couplings, $\left|Y_{i j}\right|$ and $\left|Y_{j i}\right|$ from CMS results of $\sqrt{s}=8 \mathrm{TeV}$ and $13 \mathrm{TeV}$. The different light and deep red shaded regions are restricted by the upper bounds of flavor violating LFV decays. The off-diagonal Yukawa couplings generated from holomorphic and non-holomorphic trilinear couplings are displayed in cyan and blue respectively. The black lines in each plot correspond to the most stringent limits on $Y_{i j}$ 's.

$l_{i} \rightarrow 3 l_{j}, l_{i} \rightarrow l_{j} \gamma$ and $\sqrt{\left(Y_{i j}^{2}+Y_{j i}^{2}\right)}$ in the $\left(\left|Y_{i j}\right|-\left|Y_{j i}\right|\right)$ plane. The observed LHC limits on $\sqrt{\left(Y_{i j}^{2}+Y_{j i}^{2}\right)}$ for $\sqrt{s}=13 \mathrm{TeV}$ which are derived from the direct searches of $\operatorname{Br}(h \rightarrow \mu \tau)$ and $\operatorname{Br}(h \rightarrow e \tau)$ [127] are shown as black solid curves in figure 13(b) and (c). These indeed constitute the most stringent limits concerning the 2 nd and $3 \mathrm{rd}$ generations of sleptons. As mentioned earlier, the Higgs boson going to $\mu \tau$ channel gives $\sqrt{\left(Y_{\mu \tau}^{2}+Y_{\tau \mu}^{2}\right)}<1.50 \times 10^{-3}$ and the same for $e \tau$ channel leads to $\sqrt{\left(Y_{e \tau}^{2}+Y_{\tau e}^{2}\right)}<2.26 \times 10^{-3}$ at $95 \%$ confidence level. These limits constitute a significant improvement in the $\mu \tau$ channel over the previously obtained limits by CMS and ATLAS using $8 \mathrm{TeV}$ proton-proton collision data corresponding to an integrated luminosity of about $20 \mathrm{fb}^{-1}[124,131,132]$ shown by the green curves above the black curves. For the $e \tau$ mode, in the $8 \mathrm{TeV}$ analysis, the allowed value of $\sqrt{\left(Y_{e \tau}^{2}+Y_{\tau e}^{2}\right)}$ was less than $2.4 \times 10^{-3}$, so the $13 \mathrm{TeV}$ limit is seen to be almost overlapped with the $8 \mathrm{TeV}$ one in the middle plot of figure 13 . We observe that some of the blue points originated from the non-holomorphic trilinear couplings exceed the current LHC limits but almost all the cyan points from the holomorphic couplings are safe here. Needless to mention again, all the data points shown here respect charge breaking minima condition. For the first two generations, most dominant constraint comes from the absence of $\mu \rightarrow e \gamma$, which is shown by the solid black curve in $\left|Y_{e \mu}\right|-\left|Y_{\mu e}\right|$ parameter space. One may find that, the LHC $\sqrt{s}=13 \mathrm{TeV}$ data puts $95 \%$ confidence level constraints on Yukawa couplings derived from $\operatorname{Br}(h \rightarrow e \mu)<6.2 \times 10^{-5}$ which yields $\sqrt{\left(Y_{e \mu}^{2}+Y_{\mu e}^{2}\right)}$ to be less than $2.24 \times 10^{-4}$ [132]. Absence of $\mu \rightarrow e \gamma$ regulates it even more, implying a limit of $\sqrt{\left(Y_{e \mu}^{2}+Y_{\mu e}^{2}\right)}<3.6 \times 10^{-6}$ [130]. Such a tiny Yukawa coupling, with $\cos (\beta-\alpha) \sim 10^{-3} / 10^{-4}$ leads to $\operatorname{Br}(h \rightarrow e \mu) \sim(O)\left(10^{-13}\right)$ or so. ${ }^{8}$

\footnotetext{
${ }^{8}$ Here we should state that for non-decoupling Higgs, $m_{A} \gtrsim m_{h}, \cos (\beta-\alpha)$ can be large which may enhance $\operatorname{Br}(h \rightarrow e \mu / e \tau / \mu \tau)$. For maximum $\operatorname{Br}\left(h \rightarrow l_{i} \bar{l}_{j}\right)$ one may see the reference [56].
} 


\begin{tabular}{|c|c|c|c|c|c|}
\hline Processes & Maximum BR & $\begin{array}{c}\text { Slepton Mass } \\
{[\mathrm{GeV}]}\end{array}$ & Parameter & $\begin{array}{c}\text { Maximum Ranges } \\
\text { of } A_{i j}^{\prime} \& A_{i j}[\mathrm{GeV}]\end{array}$ & $\begin{array}{c}\text { Most Sensitive } \\
\text { to }\end{array}$ \\
\hline$\mu \rightarrow e \gamma$ & $4.20 \times 10^{-13}$ & 1000 & &,$--[-100: 100]$ & \\
$\mu \rightarrow 3 e$ & $7.52 \times 10^{-15}$ & 2000 & $A_{e \mu}^{\prime}, A_{e \mu}$ & {$[-8: 8],[-350: 350]$} & Bounds from \\
$h \rightarrow e \mu$ & $5.07 \times 10^{-13}$ & 3000 & & {$[-20,20],[-800: 800]$} & $B r(\mu \rightarrow e \gamma)$ \\
$H / A \rightarrow e \mu$ & $2.5 \times 10^{-10}$ & 5000 & & {$[-50,50],[-1500: 1500]$} & \\
\hline$\tau \rightarrow e \gamma$ & $1.66 \times 10^{-9}$ & 1000 & &,$--[-3000: 3000]$ & \\
$\tau \rightarrow 3 e$ & $1.53 \times 10^{-11}$ & 2000 & $A_{e \tau}^{\prime}, A_{e \tau}$ & {$[-650: 650],[-6500: 6500]$} & LHC null results of \\
$h \rightarrow e \tau$ & $1.21 \times 10^{-10}$ & 3000 & & {$[-1400: 1400],[-8000: 8000]$} & $B r(h \rightarrow e \tau)$ \\
$H / A \rightarrow e \tau$ & $3.20 \times 10^{-5}$ & 5000 & & {$[-3800: 3800],[-8000: 8000]$} & \\
\hline$\tau \rightarrow \mu \gamma$ & $2.90 \times 10^{-9}$ & 1000 & &,$--[-2700: 2700]$ & \\
$\tau \rightarrow 3 \mu$ & $6.97 \times 10^{-12}$ & 2000 & $A_{\mu \tau}^{\prime}, A_{\mu \tau}$ & {$[-350: 350],[-6000: 6000]$} & LHC null results of \\
$h \rightarrow \mu \tau$ & $3.24 \times 10^{-10}$ & 3000 & & {$[-800: 800],[-8000: 8000]$} & $B r(h \rightarrow \mu \tau)$ \\
$H / A \rightarrow \mu \tau$ & $5.54 \times 10^{-5}$ & 5000 & & {$[-2400: 2400],[-8000: 8000]$} & \\
\hline
\end{tabular}

Table 4. Allowed ranges of holomorphic and non-holomorphic trilinear couplings and the corresponding maximum decay branching ratios after avoiding charge breaking minima, respecting the upper limits of various other LFV decays and LHC constraints on the off-diagonal Yukawa couplings from the non-observation of a scalar boson decaying into $e \mu / e \tau / \mu \tau$ channels.

One may further combine the results of figures 12 and 13 to conclusively draw upper limits on $A_{i j}^{\prime}$ 's obeying the observations related to relevant LFV processes and charge breaking minima bounds. This in turn would determine maximum allowed branching ratios for our concerned cLFV process which could be tested in the near future. For completeness we summarize our results in the table 4 which shows the allowed values of $A_{i j}^{l}$ and $A_{i j}^{\prime l}$ in general and the resulting maximum values of different LFV decay branching ratios. Our results can be summarized as follows:

(i) For first two generations of lepton, rise off-diagonal holomorphic and NH trilinear couplings i.e., $A_{e \mu}^{(\prime)}$ are visibly restricted by the upper bound of $\mu \rightarrow e \gamma$.

(ii) The other two combinations of trilinear coupling parameters, namely, $e \tau$ and $\mu \tau$ are regulated by the CMS $13 \mathrm{TeV}$ results.

(iii) Finally the derived allowed ranges for $A_{e \mu}$ and $A_{e \mu}^{\prime}$ are much more restricted compared to $A_{e \tau}^{(\prime)}$ or $A_{\mu \tau}^{(\prime)}$.

\section{Conclusion}

The Minimal Supersymmetric Standard Model when extended with the most general soft SUSY breaking trilinear terms may lead to interesting phenomenologies. These additional terms that are non-holomorphic in nature were analyzed in several studies in the past as well as in the recent years. We focus on introducing flavor violating lepton decays and Higgs decaying to charged leptons involving flavor violation due to non-vanishing 
non-diagonal entries of the trilinear coupling matrices both of standard and non-standard types. In this analysis, we first upgrade the existing analytical result involving trilinear couplings for avoiding the appearance of charge breaking minima of vacuum in MSSM. In other words, we extend the traditional analytical result for charge breaking in MSSM by including non-diagonal entries of the soft-breaking trilinear coupling matrices $\left(A_{i j}^{l}\right)$. We extend the analysis further by involving non-holomorphic trilinear coupling matrices $\left(A_{i j}^{\prime l}\right)$. By considering vevs for appropriate sleptons and non-vanishing values of $A_{i j}^{\prime l}$ we are able to delineate regions of parameter space that are associated with appearance of charge breaking minima of the vacuum. On the contrary, we also find plenty of possibilities of evading the charge breaking conditions even with reasonably large values of $A_{i j}^{\prime l}$ due to cancellation of terms in the analytical result. We studied the effects of considering nonvanishing off-diagonal trilinear terms of both types, one by one, on cLFV processes like $l_{j} \rightarrow l_{i} \gamma$ or $l_{j} \rightarrow 3 l_{i}$ and all the variants of $\operatorname{Higgs}(h, H, A)$ decays into $l_{i} \bar{l}_{j}$ involving flavor violation. For simplicity, we do not consider any flavor violation effect from the slepton mass matrices. In this phenomenological work, we include (i) the present and future experimental sensitivities of cLFV observables, and (ii) the $8 \mathrm{TeV}$ and $13 \mathrm{TeV}$ CMS results that search SM Higgs boson decays into flavor violating modes, namely e $\tau$ or $\mu \tau$. We find that $\mathrm{NH}$ couplings namely $A_{i j}^{\prime l}$ are better suited in achieving larger rates for all flavor violating decay observables that can potentially be tested in the near future. In particular, $\mu \rightarrow e \gamma$ would be more favourable to test $A_{i j}^{\prime l}$ involving first two generation sleptons. On the other hand, MSSM Higgs decays (specially that of the heavier Higgs bosons) into LFV modes may strongly be influenced by $A_{e \tau}^{\prime}$ or $A_{\mu \tau}^{\prime}$. For most of these observables the standard trilinear couplings $A_{i j}$ turn out to be inadequate to produce any significant contribution in relation to the present or future experimental measurements. This indeed emphasizes the usefulness of including the non-holomorphic trilinear terms for such analyses.

\section{Acknowledgments}

SM would like to thank Abhishek Dey for many helpful discussions. The computations was supported in part by the SAMKHYA: the High Performance Computing Facility provided by Institute of Physics, Bhubaneswar.

Open Access. This article is distributed under the terms of the Creative Commons Attribution License (CC-BY 4.0), which permits any use, distribution and reproduction in any medium, provided the original author(s) and source are credited.

\section{References}

[1] ATLAS and CMS collaborations, Measurements of the Higgs boson production and decay rates and constraints on its couplings from a combined ATLAS and CMS analysis of the LHC pp collision data at $\sqrt{s}=7$ and 8 TeV, JHEP 08 (2016) 045 [arXiv: 1606.02266] [INSPIRE]. 
[2] ATLAS collaboration, Observation of a new particle in the search for the Standard Model Higgs boson with the ATLAS detector at the LHC, Phys. Lett. B 716 (2012) 1 [arXiv:1207.7214] [INSPIRE].

[3] CMS collaboration, Observation of a new boson at a mass of $125 \mathrm{GeV}$ with the CMS experiment at the LHC, Phys. Lett. B 716 (2012) 30 [arXiv:1207.7235] [INSPIRE].

[4] S.T. Petcov, The processes $\mu \rightarrow e \gamma, \mu \rightarrow e e \bar{e}, \nu^{\prime} \rightarrow \nu \gamma$ in the Weinberg-Salam model with neutrino mixing, Sov. J. Nucl. Phys. 25 (1977) 340 [Erratum ibid. 25 (1977) 698] [Yad. Fiz. 25 (1977) 641] [Erratum ibid. 25 (1977) 1336] [InSPIRE].

[5] T.P. Cheng and L.-F. Li, Nonconservation of separate $\mu$-lepton and e-lepton numbers in gauge theories with $V+A$ currents, Phys. Rev. Lett. 38 (1977) 381 [INSPIRE].

[6] S.M. Bilenky, S.T. Petcov and B. Pontecorvo, Lepton mixing, $\mu \rightarrow e+\gamma$ decay and neutrino oscillations, Phys. Lett. B 67 (1977) 309 [INSPIRE].

[7] M. Raidal et al., Flavour physics of leptons and dipole moments, Eur. Phys. J. C 57 (2008) 13 [arXiv:0801.1826] [INSPIRE].

[8] H.P. Nilles, Supersymmetry, supergravity and particle physics, Phys. Rept. 110 (1984) 1 [INSPIRE].

[9] J.D. Lykken, Introduction to supersymmetry, in Fields, strings and duality. Proceedings, Summer School, Theoretical Advanced Study Institute in Elementary Particle Physics, TASI'96, Boulder, CO, U.S.A., 2-28 June 1996, pg. 85 [hep-th/9612114] [INSPIRE].

[10] J. Wess and J. Bagger, Supersymmetry and supergravity, second edition, Princeton University Press, Princeton, NJ, U.S.A. (1991).

[11] M. Drees, P. Roy and R.M. Godbole, Theory and phenomenology of sparticles, World Scientific, Singapore (2005).

[12] H. Baer and X. Tata, Weak scale supersymmetry: from superfields to scattering events, Cambridge Univ. Pr., Cambridge, U.K. (2006).

[13] D.J.H. Chung, L.L. Everett, G.L. Kane, S.F. King, J.D. Lykken and L.-T. Wang, The soft supersymmetry breaking Lagrangian: theory and applications, Phys. Rept. 407 (2005) 1 [hep-ph/0312378] [INSPIRE].

[14] H.E. Haber and G.L. Kane, The search for supersymmetry: probing physics beyond the Standard Model, Phys. Rept. 117 (1985) 75 [INSPIRE].

[15] S.P. Martin, A supersymmetry primer, hep-ph/9709356 [INSPIRE].

[16] F. Gabbiani, E. Gabrielli, A. Masiero and L. Silvestrini, A complete analysis of FCNC and CP constraints in general SUSY extensions of the Standard Model, Nucl. Phys. B 477 (1996) 321 [hep-ph/9604387] [INSPIRE].

[17] P.F. de Salas, D.V. Forero, C.A. Ternes, M. Tortola and J.W.F. Valle, Status of neutrino oscillations 2018: $3 \sigma$ hint for normal mass ordering and improved CP sensitivity, Phys. Lett. B 782 (2018) 633 [arXiv: 1708.01186] [INSPIRE].

[18] I. Esteban, M.C. Gonzalez-Garcia, M. Maltoni, I. Martinez-Soler and T. Schwetz, Updated fit to three neutrino mixing: exploring the accelerator-reactor complementarity, JHEP 01 (2017) 087 [arXiv: 1611.01514] [inSPIRE].

[19] P. Minkowski, $\mu \rightarrow$ er at a rate of one out of $10^{9}$ muon decays?, Phys. Lett. B 67 (1977) 421 [INSPIRE]. 
[20] M. Gell-Mann, P. Ramond and R. Slansky, Complex spinors and unified theories, in Supergravity, P. Van Nieuwenhuizen and D.Z. Freedman eds., North-Holland, Amsterdam, The Netherlands (1979), pg. 315 [PRINT-80-0576] [Conf. Proc. C 790927 (1979) 315] [arXiv:1306 .4669] [INSPIRE].

[21] T. Yanagida, Horizontal gauge symmetry and masses of neutrinos, in Proceedings of the workshop on the unified theory and the baryon number in the universe, O. Sawada and A. Sugamoto eds., KEK, Tsukuba, Japan (1979), pg. 95 [InSPIRE].

[22] S.L. Glashow, The future of elementary particle physics, in Quarks and leptons, M. Lévy et al. eds., Plenum Press, New York, NY, U.S.A. (1980), pg. 687 [NATO Sci. Ser. B 61 (1980) 687] [INSPIRE].

[23] R.N. Mohapatra and G. Senjanović, Neutrino mass and spontaneous parity nonconservation, Phys. Rev. Lett. 44 (1980) 912 [INSPIRE].

[24] R. Barbieri, D.V. Nanopoulos, G. Morchio and F. Strocchi, Neutrino masses in grand unified theories, Phys. Lett. B 90 (1980) 91 [INSPIRE].

[25] R.E. Marshak and R.N. Mohapatra, Selection rules for baryon number nonconservation in gauge models, invited talk given at Orbis Scientiae, Coral Gables, FL, U.S.A. 14-17 January 1980 [INSPIRE].

[26] T.P. Cheng and L.-F. Li, Neutrino masses, mixings and oscillations in $\mathrm{SU}(2) \times \mathrm{U}(1)$ models of electroweak interactions, Phys. Rev. D 22 (1980) 2860 [INSPIRE].

[27] M. Magg and C. Wetterich, Neutrino mass problem and gauge hierarchy, Phys. Lett. B 94 (1980) 61 [INSPIRE].

[28] G. Lazarides, Q. Shafi and C. Wetterich, Proton lifetime and fermion masses in an $\mathrm{SO}(10)$ model, Nucl. Phys. B 181 (1981) 287 [INSPIRE].

[29] J. Schechter and J.W.F. Valle, Neutrino masses in $\mathrm{SU}(2) \times \mathrm{U}(1)$ theories, Phys. Rev. D 22 (1980) 2227 [INSPIRE].

[30] R.N. Mohapatra and G. Senjanović, Neutrino masses and mixings in gauge models with spontaneous parity violation, Phys. Rev. D 23 (1981) 165 [inSPIRE].

[31] E. Ma, Pathways to naturally small neutrino masses, Phys. Rev. Lett. 81 (1998) 1171 [hep-ph/9805219] [INSPIRE].

[32] R. Foot, H. Lew, X.G. He and G.C. Joshi, Seesaw neutrino masses induced by a triplet of leptons, Z. Phys. C 44 (1989) 441 [inSPIRE].

[33] S.F. King, Atmospheric and solar neutrinos with a heavy singlet, Phys. Lett. B 439 (1998) 350 [hep-ph/9806440] [INSPIRE].

[34] S. Davidson and S.F. King, Bimaximal neutrino mixing in the MSSM with a single right-handed neutrino, Phys. Lett. B 445 (1998) 191 [hep-ph/9808296] [INSPIRE].

[35] F. Borzumati and A. Masiero, Large muon and electron number violations in supergravity theories, Phys. Rev. Lett. 57 (1986) 961 [INSPIRE].

[36] J. Hisano, T. Moroi, K. Tobe and M. Yamaguchi, Lepton flavor violation via right-handed neutrino Yukawa couplings in supersymmetric Standard Model, Phys. Rev. D 53 (1996) 2442 [hep-ph/9510309] [INSPIRE]. 
[37] J. Hisano, T. Moroi, K. Tobe, M. Yamaguchi and T. Yanagida, Lepton flavor violation in the supersymmetric Standard Model with seesaw induced neutrino masses, Phys. Lett. B 357 (1995) 579 [hep-ph/9501407] [INSPIRE].

[38] J. Hisano and D. Nomura, Solar and atmospheric neutrino oscillations and lepton flavor violation in supersymmetric models with the right-handed neutrinos, Phys. Rev. D 59 (1999) 116005 [hep-ph/9810479] [INSPIRE].

[39] C. Hamzaoui, M. Pospelov and M. Toharia, Higgs mediated FCNC in supersymmetric models with large $\tan \beta$, Phys. Rev. D 59 (1999) 095005 [hep-ph/9807350] [InSPIRE].

[40] J.L. Diaz-Cruz and J.J. Toscano, Lepton flavor violating decays of Higgs bosons beyond the Standard Model, Phys. Rev. D 62 (2000) 116005 [hep-ph/9910233] [INSPIRE].

[41] G. Isidori and A. Retico, Scalar flavor changing neutral currents in the large $\tan \beta$ limit, JHEP 11 (2001) 001 [hep-ph/0110121] [InSPIRE].

[42] K.S. Babu and C. Kolda, Higgs mediated $\tau \rightarrow 3 \mu$ in the supersymmetric seesaw model, Phys. Rev. Lett. 89 (2002) 241802 [hep-ph/0206310] [INSPIRE].

[43] A. Masiero, S.K. Vempati and O. Vives, Seesaw and lepton flavor violation in SUSY SO(10), Nucl. Phys. B 649 (2003) 189 [hep-ph/0209303] [InSPIRE].

[44] M. Ciuchini, A. Masiero, L. Silvestrini, S.K. Vempati and O. Vives, Grand unification of quark and lepton FCNCs, Phys. Rev. Lett. 92 (2004) 071801 [hep-ph/0307191] [INSPIRE].

[45] A. Masiero, S.K. Vempati and O. Vives, SUSY seesaw and FCNC, Nucl. Phys. Proc. Suppl. 137 (2004) 156 [hep-ph/0405017] [INSPIRE].

[46] A. Masiero, S.K. Vempati and O. Vives, Massive neutrinos and flavor violation, New J. Phys. 6 (2004) 202 [hep-ph/0407325] [INSPIRE].

[47] L. Calibbi, A. Faccia, A. Masiero and S.K. Vempati, Lepton flavour violation from SUSY-GUTs: where do we stand for MEG, PRISM/PRIME and a super flavour factory, Phys. Rev. D 74 (2006) 116002 [hep-ph/0605139] [inSPIRE].

[48] P. Paradisi, Higgs-mediated $\tau \rightarrow \mu$ and $\tau \rightarrow e$ transitions in II Higgs doublet model and supersymmetry, JHEP 02 (2006) 050 [hep-ph/0508054] [INSPIRE].

[49] A. Brignole and A. Rossi, Lepton flavor violating decays of supersymmetric Higgs bosons, Phys. Lett. B 566 (2003) 217 [hep-ph/0304081] [INSPIRE].

[50] A. Brignole and A. Rossi, Anatomy and phenomenology of $\mu-\tau$ lepton flavor violation in the MSSM, Nucl. Phys. B 701 (2004) 3 [hep-ph/0404211] [INSPIRE].

[51] T. Han and D. Marfatia, $h \rightarrow \mu \tau$ at hadron colliders, Phys. Rev. Lett. 86 (2001) 1442 [hep-ph/0008141] [INSPIRE].

[52] E. Arganda, A.M. Curiel, M.J. Herrero and D. Temes, Lepton flavor violating Higgs boson decays from massive seesaw neutrinos, Phys. Rev. D 71 (2005) 035011 [hep-ph/0407302] [INSPIRE].

[53] E. Arganda and M.J. Herrero, Testing supersymmetry with lepton flavor violating $\tau$ and $\mu$ decays, Phys. Rev. D 73 (2006) 055003 [hep-ph/0510405] [INSPIRE].

[54] M.E. Gomez, S. Heinemeyer and M. Rehman, Effects of sfermion mixing induced by RGE running in the minimal flavor violating CMSSM, Eur. Phys. J. C 75 (2015) 434 [arXiv: 1501.02258] [INSPIRE]. 
[55] M.E. Gomez, S. Heinemeyer and M. Rehman, Lepton flavor violating Higgs boson decays in supersymmetric high scale seesaw models, arXiv:1703.02229 [INSPIRE].

[56] D. Aloni, Y. Nir and E. Stamou, Large $\mathrm{BR}(h \rightarrow \tau \mu)$ in the MSSM?, JHEP 04 (2016) 162 [arXiv: 1511.00979] [INSPIRE].

[57] A. Arhrib, Y. Cheng and O.C.W. Kong, Comprehensive analysis on lepton flavor violating Higgs boson to $\mu^{\mp} \tau^{ \pm}$decay in supersymmetry without $R$ parity, Phys. Rev. D 87 (2013) 015025 [arXiv: 1210.8241] [INSPIRE].

[58] M. Arana-Catania, E. Arganda and M.J. Herrero, Non-decoupling SUSY in LFV Higgs decays: a window to new physics at the LHC, JHEP 09 (2013) 160 [Erratum ibid. 10 (2015) 192] [arXiv: 1304.3371] [INSPIRE].

[59] A. Abada, M.E. Krauss, W. Porod, F. Staub, A. Vicente and C. Weiland, Lepton flavor violation in low-scale seesaw models: SUSY and non-SUSY contributions, JHEP 11 (2014) 048 [arXiv: 1408.0138] [INSPIRE].

[60] A. Hammad, S. Khalil and C.S. Un, Large $\mathrm{BR}(h \rightarrow \tau \mu)$ in supersymmetric models, Phys. Rev. D 95 (2017) 055028 [arXiv: 1605.07567] [INSPIRE].

[61] J.L. Evans, K. Kadota and T. Kuwahara, Revisiting flavor and CP-violation in supersymmetric SU(5) with right-handed neutrinos, Phys. Rev. D 98 (2018) 075030 [arXiv: 1807.08234] [INSPIRE].

[62] F. Deppisch and J.W.F. Valle, Enhanced lepton flavor violation in the supersymmetric inverse seesaw model, Phys. Rev. D 72 (2005) 036001 [hep-ph/0406040] [INSPIRE].

[63] A. Abada, D. Das and C. Weiland, Enhanced Higgs mediated lepton flavour violating processes in the supersymmetric inverse seesaw model, JHEP 03 (2012) 100 [arXiv:1111.5836] [INSPIRE].

[64] A. Abada, D. Das, A. Vicente and C. Weiland, Enhancing lepton flavour violation in the supersymmetric inverse seesaw beyond the dipole contribution, JHEP 09 (2012) 015 [arXiv:1206.6497] [INSPIRE].

[65] E. Arganda, M.J. Herrero, X. Marcano and C. Weiland, Imprints of massive inverse seesaw model neutrinos in lepton flavor violating Higgs boson decays, Phys. Rev. D 91 (2015) 015001 [arXiv: 1405.4300] [INSPIRE].

[66] E. Arganda, M.J. Herrero, X. Marcano and C. Weiland, Enhancement of the lepton flavor violating Higgs boson decay rates from SUSY loops in the inverse seesaw model, Phys. Rev. D 93 (2016) 055010 [arXiv: 1508.04623] [INSPIRE].

[67] E. Arganda, M.J. Herrero, R. Morales and A. Szynkman, Analysis of the $h, H, A \rightarrow \tau \mu$ decays induced from SUSY loops within the mass insertion approximation, JHEP 03 (2016) 055 [arXiv: 1510.04685$]$ [INSPIRE].

[68] R. Barbieri and L.J. Hall, Signals for supersymmetric unification, Phys. Lett. B 338 (1994) 212 [hep-ph/9408406] [INSPIRE].

[69] R. Barbieri, L.J. Hall and A. Strumia, Violations of lepton flavor and CP in supersymmetric unified theories, Nucl. Phys. B 445 (1995) 219 [hep-ph/9501334] [INSPIRE].

[70] L.J. Hall, V.A. Kostelecky and S. Raby, New flavor violations in supergravity models, Nucl. Phys. B 267 (1986) 415 [INSPIRE]. 
[71] P.S.B. Dev and R.N. Mohapatra, TeV scale inverse seesaw in $\mathrm{SO}(10)$ and leptonic non-unitarity effects, Phys. Rev. D 81 (2010) 013001 [arXiv:0910.3924] [INSPIRE].

[72] A. Brignole, L.E. Ibáñez and C. Muñoz, Soft supersymmetry breaking terms from supergravity and superstring models, Adv. Ser. Direct. High Energy Phys. 18 (1998) 125 [hep-ph/9707209] [INSPIRE].

[73] I. Jack and D.R.T. Jones, Nonstandard soft supersymmetry breaking, Phys. Lett. B 457 (1999) 101 [hep-ph/9903365] [INSPIRE].

[74] S.P. Martin, Dimensionless supersymmetry breaking couplings, flat directions and the origin of intermediate mass scales, Phys. Rev. D 61 (2000) 035004 [hep-ph/9907550] [INSPIRE].

[75] I. Jack and D.R.T. Jones, Quasiinfrared fixed points and renormalization group invariant trajectories for nonholomorphic soft supersymmetry breaking, Phys. Rev. D 61 (2000) 095002 [hep-ph/9909570] [INSPIRE].

[76] H.E. Haber and J.D. Mason, Hard supersymmetry-breaking 'wrong-Higgs' couplings of the MSSM, Phys. Rev. D 77 (2008) 115011 [arXiv:0711.2890] [INSPIRE].

[77] J.P.J. Hetherington, The spectrum of the MSSM with nonstandard supersymmetry breaking, JHEP 10 (2001) 024 [hep-ph/0108206] [INSPIRE].

[78] U. Chattopadhyay and A. Dey, Probing non-holomorphic MSSM via precision constraints, dark matter and LHC data, JHEP 10 (2016) 027 [arXiv: 1604.06367] [INSPIRE].

[79] C.S. Ün, Ş.H. Tanyıldızı, S. Kerman and L. Solmaz, Generalized soft breaking leverage for the MSSM, Phys. Rev. D 91 (2015) 105033 [arXiv:1412.1440] [INSPIRE].

[80] G.G. Ross, K. Schmidt-Hoberg and F. Staub, On the MSSM Higgsino mass and fine tuning, Phys. Lett. B 759 (2016) 110 [arXiv:1603.09347] [INSPIRE].

[81] G.G. Ross, K. Schmidt-Hoberg and F. Staub, Revisiting fine-tuning in the MSSM, JHEP 03 (2017) 021 [arXiv: 1701.03480] [INSPIRE].

[82] U. Chattopadhyay, D. Das and S. Mukherjee, Exploring non-holomorphic soft terms in the framework of gauge mediated supersymmetry breaking, JHEP 01 (2018) 158 [arXiv: 1710.10120] [INSPIRE].

[83] A. Crivellin, Z. Fabisiewicz, W. Materkowska, U. Nierste, S. Pokorski and J. Rosiek, Lepton flavour violation in the MSSM: exact diagonalization vs mass expansion, JHEP 06 (2018) 003 [arXiv: 1802.06803] [INSPIRE].

[84] A. Crivellin, L. Hofer and J. Rosiek, Complete resummation of chirally-enhanced loop-effects in the MSSM with non-minimal sources of flavor-violation, JHEP 07 (2011) 017 [arXiv:1103.4272] [INSPIRE].

[85] J. Rosiek, Complete set of Feynman rules for the MSSM: erratum, hep-ph/9511250 [INSPIRE].

[86] X.-Y. Pham, Lepton flavor changing in neutrinoless tau decays, Eur. Phys. J. C 8 (1999) 513 [hep-ph/9810484] [INSPIRE].

[87] BABAR collaboration, Limits on tau lepton-flavor violating decays in three charged leptons, Phys. Rev. D 81 (2010) 111101 [arXiv:1002.4550] [INSPIRE].

[88] K. Hayasaka et al., Search for lepton flavor violating $\tau$ decays into three leptons with 719 million produced $\tau^{+} \tau^{-}$pairs, Phys. Lett. B 687 (2010) 139 [arXiv:1001.3221] [INSPIRE]. 
[89] LHCb collaboration, Search for the lepton flavour violating decay $\tau^{-} \rightarrow \mu^{-} \mu^{+} \mu^{-}$, JHEP 02 (2015) 121 [arXiv: 1409.8548] [INSPIRE].

[90] Heavy Flavor Averaging Group (HFAG) collaboration, Averages of b-hadron, c-hadron and $\tau$-lepton properties as of Summer 2014, arXiv:1412.7515 [INSPIRE].

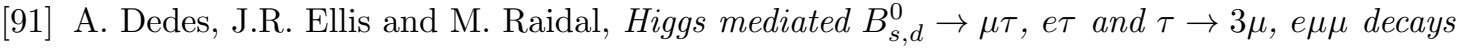
in supersymmetric seesaw models, Phys. Lett. B 549 (2002) 159 [hep-ph/0209207] [INSPIRE].

[92] D. Choudhury, F. Eberlein, A. Konig, J. Louis and S. Pokorski, Constraints on nonuniversal soft terms from flavor changing neutral currents, Phys. Lett. B 342 (1995) 180 [hep-ph/9408275] [INSPIRE].

[93] J.A. Casas and S. Dimopoulos, Stability bounds on flavor violating trilinear soft terms in the MSSM, Phys. Lett. B 387 (1996) 107 [hep-ph/9606237] [INSPIRE].

[94] J.M. Frere, D.R.T. Jones and S. Raby, Fermion masses and induction of the weak scale by supergravity, Nucl. Phys. B 222 (1983) 11 [INSPIRE].

[95] J.A. Casas, A. Lleyda and C. Muñoz, Strong constraints on the parameter space of the MSSM from charge and color breaking minima, Nucl. Phys. B 471 (1996) 3 [hep-ph/9507294] [INSPIRE].

[96] J.F. Gunion, H.E. Haber and M. Sher, Charge/color breaking minima and A-parameter bounds in supersymmetric models, Nucl. Phys. B 306 (1988) 1 [INSPIRE].

[97] M. Drees, M. Gluck and K. Grassie, A new class of false vacua in low-energy $N=1$ supergravity theories, Phys. Lett. B 157 (1985) 164 [INSPIRE].

[98] H. Komatsu, New constraints on parameters in the minimal supersymmetric model, Phys. Lett. B 215 (1988) 323 [INSPIRE].

[99] P. Langacker and N. Polonsky, Implications of Yukawa unification for the Higgs sector in supersymmetric grand unified models, Phys. Rev. D 50 (1994) 2199 [hep-ph/9403306] [INSPIRE].

[100] A. Strumia, Charge and color breaking minima and constraints on the MSSM parameters, Nucl. Phys. B 482 (1996) 24 [hep-ph/9604417] [INSPIRE].

[101] W.G. Hollik, A new view on vacuum stability in the MSSM, JHEP 08 (2016) 126 [arXiv: 1606.08356] [INSPIRE].

[102] U. Chattopadhyay and A. Dey, Exploring MSSM for charge and color breaking and other constraints in the context of Higgs@125 GeV, JHEP 11 (2014) 161 [arXiv:1409.0611] [INSPIRE].

[103] D. Chowdhury, R.M. Godbole, K.A. Mohan and S.K. Vempati, Charge and color breaking constraints in MSSM after the Higgs discovery at LHC, JHEP 02 (2014) 110 [Erratum ibid. 03 (2018) 149] [arXiv:1310.1932] [INSPIRE].

[104] J. Beuria and A. Dey, Exploring charge and color breaking vacuum in non-holomorphic MSSM, JHEP 10 (2017) 154 [arXiv:1708.08361] [INSPIRE].

[105] A. Kusenko, P. Langacker and G. Segre, Phase transitions and vacuum tunneling into charge and color breaking minima in the MSSM, Phys. Rev. D 54 (1996) 5824 [hep-ph/9602414] [INSPIRE]. 
[106] A. Kusenko and P. Langacker, Is the vacuum stable?, Phys. Lett. B 391 (1997) 29 [hep-ph/9608340] [INSPIRE].

[107] A. Kusenko, Color and charge breaking minima in the MSSM, Nucl. Phys. Proc. Suppl. 52A (1997) 67 [hep-ph/9607287] [INSPIRE].

[108] A. Kusenko, Improved action method for analyzing tunneling in quantum field theory, Phys. Lett. B 358 (1995) 51 [hep-ph/9504418] [INSPIRE].

[109] R.H. Brandenberger, Quantum field theory methods and inflationary universe models, Rev. Mod. Phys. 57 (1985) 1 [INSPIRE].

[110] C. Le Mouel, Charge and color breaking conditions associated to the top quark Yukawa coupling, Phys. Rev. D 64 (2001) 075009 [hep-ph/0103341] [INSPIRE].

[111] U. Chattopadhyay, A. Datta, S. Mukherjee and A.K. Swain, Sbottoms as probes to MSSM with nonholomorphic soft interactions, JHEP 10 (2018) 202 [arXiv: 1809.05438] [INSPIRE].

[112] MEG collaboration, Search for the lepton flavour violating decay $\mu^{+} \rightarrow e^{+} \gamma$ with the full dataset of the MEG experiment, Eur. Phys. J. C 76 (2016) 434 [arXiv:1605.05081] [INSPIRE].

[113] A.M. Baldini et al., MEG upgrade proposal, arXiv:1301.7225 [INSPIRE].

[114] A. Blondel et al., Research proposal for an experiment to search for the decay $\mu \rightarrow$ eee, arXiv: 1301.6113 [INSPIRE].

[115] Mu3E collaboration, Status of the Mu3e experiment at PSI, EPJ Web Conf. 118 (2016) 01028 [arXiv: 1605. 02906] [INSPIRE].

[116] SINDRUM collaboration, Search for the decay $\mu^{+} \rightarrow e^{+} e^{+} e^{-}$, Nucl. Phys. B 299 (1988) 1 [INSPIRE].

[117] Mu3E collaboration, The rare and forbidden: testing physics beyond the Standard Model with Mu3e, SciPost Phys. Proc. 1 (2019) 052 [arXiv: 1812.00741] [InSPIRE].

[118] Belle collaboration, New search for $\tau \rightarrow \mu \gamma$ and $\tau \rightarrow e \gamma$ decays at Belle, Phys. Lett. B 666 (2008) 16 [arXiv:0705.0650] [inSPIRE].

[119] BABAR collaboration, Searches for lepton flavor violation in the decays $\tau^{ \pm} \rightarrow e^{ \pm} \gamma$ and $\tau^{ \pm} \rightarrow \mu^{ \pm} \gamma$, Phys. Rev. Lett. 104 (2010) 021802 [arXiv:0908.2381] [INSPIRE].

[120] LHCb collaboration, Searches for violation of lepton flavour and baryon number in $\tau$ lepton decays at LHCb, Phys. Lett. B 724 (2013) 36 [arXiv:1304.4518] [INSPIRE].

[121] T. Aushev et al., Physics at Super B factory, arXiv:1002.5012 [INSPIRE].

[122] BeLLE collaboration, Search for lepton-flavor-violating tau decay and lepton-number-violation B decay at Belle, PoS(ICHEP2010)241 (2010) [arXiv:1011.6474] [INSPIRE].

[123] SUPERB collaboration, Super B progress reports - physics, arXiv:1008.1541 [INSPIRE].

[124] CMS collaboration, Search for lepton-flavour-violating decays of the Higgs boson, Phys. Lett. B 749 (2015) 337 [arXiv: 1502.07400] [INSPIRE].

[125] ATLAS collaboration, Search for lepton-flavour-violating $H \rightarrow \mu \tau$ decays of the Higgs boson with the ATLAS detector, JHEP 11 (2015) 211 [arXiv:1508.03372] [INSPIRE].

[126] CMS collaboration, Search for lepton flavour violating decays of the Higgs boson in the $\mu-\tau$ final state at $13 \mathrm{TeV}$, CMS-PAS-HIG-16-005, CERN, Geneva, Switzerland (2016). 
[127] CMS collaboration, Search for lepton flavour violating decays of the Higgs boson to $\mu \tau$ and eT in proton-proton collisions at $\sqrt{s}=13 \mathrm{TeV}, \mathrm{JHEP} 06$ (2018) 001 [arXiv:1712.07173] [INSPIRE].

[128] ATLAS collaboration, Searches for lepton-flavour-violating decays of the Higgs boson in $\sqrt{s}=13$ TeV pp collisions with the ATLAS detector, Phys. Lett. B 800 (2020) 135069 [arXiv: 1907.06131] [INSPIRE].

[129] Particle Data Group collaboration, Review of particle physics, Phys. Rev. D 98 (2018) 030001 [INSPIRE].

[130] R. Harnik, J. Kopp and J. Zupan, Flavor violating Higgs decays, JHEP 03 (2013) 026 [arXiv: 1209.1397] [INSPIRE].

[131] CMS collaboration, Search for lepton flavour violating decays of the Higgs boson to eT and e $\mu$ in proton-proton collisions at $\sqrt{s}=8 \mathrm{TeV}$, Phys. Lett. B 763 (2016) 472

[arXiv: 1607.03561] [INSPIRE].

[132] ATLAS collaboration, Search for the Higgs boson decays $H \rightarrow e e$ and $H \rightarrow e \mu$ in pp collisions at $\sqrt{s}=13 \mathrm{TeV}$ with the ATLAS detector, Phys. Lett. B 801 (2020) 135148 [arXiv: 1909.10235] [INSPIRE].

[133] ATLAS collaboration, Combined search for the Standard Model Higgs boson in pp collisions at $\sqrt{s}=7 \mathrm{TeV}$ with the ATLAS detector, Phys. Rev. D 86 (2012) 032003 [arXiv: 1207.0319] [INSPIRE].

[134] ATLAS collaboration, Search for the neutral Higgs bosons of the minimal supersymmetric Standard Model in pp collisions at $\sqrt{s}=7 \mathrm{TeV}$ with the ATLAS detector, JHEP 02 (2013) 095 [arXiv: 1211.6956] [INSPIRE].

[135] CMS collaboration, Evidence for the $125 \mathrm{GeV}$ Higgs boson decaying to a pair of $\tau$ leptons, JHEP 05 (2014) 104 [arXiv: 1401.5041] [INSPIRE].

[136] ATLAS collaboration, Evidence for the Higgs-boson Yukawa coupling to $\tau$ leptons with the ATLAS detector, JHEP 04 (2015) 117 [arXiv:1501.04943] [INSPIRE].

[137] CMS collaboration, Observation of the Higgs boson decay to a pair of $\tau$ leptons with the CMS detector, Phys. Lett. B 779 (2018) 283 [arXiv:1708.00373] [INSPIRE].

[138] ATLAS collaboration, Search for additional heavy neutral Higgs and gauge bosons in the ditau final state produced in $36 \mathrm{fb}^{-1}$ of pp collisions at $\sqrt{\mathrm{s}}=13 \mathrm{TeV}$ with the ATLAS detector, JHEP 01 (2018) 055 [arXiv:1709.07242] [INSPIRE].

[139] CMS collaboration, Search for additional neutral MSSM Higgs bosons in the $\tau \tau$ final state in proton-proton collisions at $\sqrt{s}=13 \mathrm{TeV}$, JHEP 09 (2018) 007 [arXiv:1803.06553] [INSPIRE].

[140] CMS collaboration, Search for neutral MSSM Higgs bosons decaying to a pair of $\tau$ leptons in pp collisions, JHEP 10 (2014) 160 [arXiv:1408.3316] [INSPIRE].

[141] ATLAS collaboration, Search for neutral Higgs bosons of the minimal supersymmetric Standard Model in pp collisions at $\sqrt{s}=8 \mathrm{TeV}$ with the ATLAS detector, JHEP 11 (2014) 056 [arXiv: 1409.6064] [INSPIRE].

[142] E. Arganda, X. Marcano, N.I. Mileo, R.A. Morales and A. Szynkman, Model-independent search strategy for the lepton-flavor-violating heavy Higgs boson decay to $\tau \mu$ at the LHC, Eur. Phys. J. C 79 (2019) 738 [arXiv:1906.08282] [INSPIRE]. 
[143] ATLAS collaboration, Search for lepton-flavour-violating decays of the Higgs and $Z$ bosons with the ATLAS detector, Eur. Phys. J. C 77 (2017) 70 [arXiv:1604.07730] [InSPIRE].

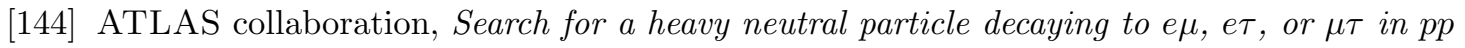
collisions at $\sqrt{s}=8 \mathrm{TeV}$ with the ATLAS detector, Phys. Rev. Lett. 115 (2015) 031801 [arXiv: 1503.04430] [INSPIRE].

[145] ATLAS collaboration, Search for lepton-flavor violation in different-flavor, high-mass final states in pp collisions at $\sqrt{s}=13 \mathrm{TeV}$ with the ATLAS detector, Phys. Rev. D 98 (2018) 092008 [arXiv: 1807.06573] [INSPIRE].

[146] LHCb collaboration, Search for lepton-flavour-violating decays of Higgs-like bosons, Eur. Phys. J. C 78 (2018) 1008 [arXiv: 1808.07135] [INSPIRE].

[147] F. Staub, SARAH 4: a tool for (not only SUSY) model builders, Comput. Phys. Commun. 185 (2014) 1773 [arXiv: 1309.7223] [INSPIRE].

[148] F. Staub, Exploring new models in all detail with SARAH, Adv. High Energy Phys. 2015 (2015) 840780 [arXiv: 1503.04200] [inSPIRE].

[149] W. Porod and F. Staub, SPheno 3.1: extensions including flavour, CP-phases and models beyond the MSSM, Comput. Phys. Commun. 183 (2012) 2458 [arXiv:1104.1573] [INSPIRE].

[150] Particle Data Group collaboration, Review of particle physics, Chin. Phys. C 40 (2016) 100001 [INSPIRE].

[151] G. Degrassi, S. Heinemeyer, W. Hollik, P. Slavich and G. Weiglein, Towards high precision predictions for the MSSM Higgs sector, Eur. Phys. J. C 28 (2003) 133 [hep-ph/0212020] [INSPIRE].

[152] B.C. Allanach, A. Djouadi, J.L. Kneur, W. Porod and P. Slavich, Precise determination of the neutral Higgs boson masses in the MSSM, JHEP 09 (2004) 044 [hep-ph/0406166] [INSPIRE].

[153] S.P. Martin, Three-loop corrections to the lightest Higgs scalar boson mass in supersymmetry, Phys. Rev. D 75 (2007) 055005 [hep-ph/0701051] [INSPIRE].

[154] R.V. Harlander, P. Kant, L. Mihaila and M. Steinhauser, Higgs boson mass in supersymmetry to three loops, Phys. Rev. Lett. 100 (2008) 191602 [arXiv:0803.0672] [INSPIRE].

[155] S. Heinemeyer, O. Stal and G. Weiglein, Interpreting the LHC Higgs search results in the MSSM, Phys. Lett. B 710 (2012) 201 [arXiv:1112.3026] [INSPIRE].

[156] A. Arbey, M. Battaglia, A. Djouadi and F. Mahmoudi, The Higgs sector of the phenomenological MSSM in the light of the Higgs boson discovery, JHEP 09 (2012) 107 [arXiv:1207.1348] [INSPIRE].

[157] M. Chakraborti, U. Chattopadhyay and R.M. Godbole, Implication of a Higgs boson at $125 \mathrm{GeV}$ within the stochastic superspace framework, Phys. Rev. D 87 (2013) 035022 [arXiv:1211.1549] [INSPIRE].

[158] ATLAS collaboration, Search for a scalar partner of the top quark in the jets plus missing transverse momentum final state at $\sqrt{s}=13$ TeV with the ATLAS detector, JHEP 12 (2017) 085 [arXiv: 1709. 04183] [inSPIRE]. 
[159] M. Sher, $\tau \rightarrow \mu \eta$ in supersymmetric models, Phys. Rev. D 66 (2002) 057301 [hep-ph/0207136] [INSPIRE].

[160] ATLAS collaboration, Search for minimal supersymmetric Standard Model Higgs bosons $H / A$ and for a $Z^{\prime}$ boson in the $\tau \tau$ final state produced in pp collisions at $\sqrt{s}=13 \mathrm{TeV}$ with the ATLAS detector, Eur. Phys. J. C 76 (2016) 585 [arXiv:1608.00890] [INSPIRE].

[161] L. Calibbi and G. Signorelli, Charged lepton flavour violation: an experimental and theoretical introduction, Riv. Nuovo Cim. 41 (2018) 71 [arXiv:1709.00294] [INSPIRE].

[162] CMS collaboration, Search for lepton flavour violating decays of a neutral heavy Higgs boson to $\mu \tau$ and e $\tau$ in proton-proton collisions at $\sqrt{s}=13 \mathrm{TeV}$, JHEP 03 (2020) 103 [arXiv: 1911.10267] [INSPIRE]. 